\title{
Immune cell repertoires in breast cancer patients after adjuvant chemotherapy
}

\author{
Claire E. Gustafson, ${ }^{1,2}$ Rohit Jadhav, ${ }^{1,2}$ Wenqiang Cao, ${ }^{1,2}$ Qian Qii, ${ }^{1,2}$ Mark Pegram, ${ }^{3}$ Lu Tian, ${ }^{4}$ \\ Cornelia M. Weyand, ${ }^{1,2}$ and Jorg J. Goronzy ${ }^{1,2}$ \\ 'Division of Immunology and Rheumatology, Department of Medicine, Stanford University School of Medicine, Stanford, \\ California, USA. ${ }^{2}$ Department of Medicine, Veterans Administration Healthcare System, Palo Alto, California, USA. \\ ${ }^{3}$ Stanford Comprehensive Cancer Institute and ${ }^{4}$ Department of Biomedical Data Science, Stanford University School of \\ Medicine, Stanford, California, USA.
}

Adjuvant chemotherapy in breast cancer patients causes immune cell depletion at an age when the regenerative capacity is compromised. Successful regeneration requires the recovery of both quantity and quality of immune cell subsets. Although immune cell numbers rebound within a year after treatment, it is unclear whether overall compositional diversity is recovered. We investigated the regeneration of immune cell complexity by comparing peripheral blood mononuclear cells from breast cancer patients ranging from 1-5 years after chemotherapy with those of age-matched healthy controls using mass cytometry and $\mathrm{T}$ cell receptor sequencing. These data reveal universal changes in patients' $\mathrm{CD}^{+} \mathrm{T}$ cells that persisted for years and consisted of expansion of Th17-like CD4 memory populations with incomplete recovery of CD4+ naive $\mathrm{T}$ cells. Conversely, $\mathrm{CD} 8^{+} \mathrm{T}$ cells fully recovered within a year. Mechanisms of T cell regeneration, however, were unbiased, as CD4+ and $C D 8^{+} \mathrm{T}$ cell receptor diversity remained high. Likewise, terminal differentiated effector memory cells were not expanded, indicating that regeneration was not driven by recognition of latent viruses. These data suggest that, while $\mathrm{CD8}^{+} \mathrm{T}$ cell immunity is successfully regenerated, the CD4 compartment may be irreversibly affected. Moreover, the bias of CD4 memory toward inflammatory effector cells may impact responses to vaccination and infection.

Conflict of interest: The authors have declared that no conflict of interest exists.

Copyright: (c) 2020, American Society for Clinical Investigation.

Submitted: October 29, 2019

Accepted: January 29, 2020

Published: February 27, 2020

Reference information: /CI Insight. 2020;5(4):e134569.

https://doi.org/10.1172/jici.

insight.134569.

\section{Introduction}

With improving cancer survival rates, the number of individuals with a history of chemotherapy treatment is steadily increasing. Indeed, there are currently more than 3 million women with a past or present history of breast cancer in the United States, many of whom have received adjuvant chemotherapy. This treatment, although highly effective, causes a massive depletion of cells in the immune system, including different types of lymphocytes such as NK cells, B cells, and T cells, at an age when the de novo generation of lymphocytes, particularly $\mathrm{T}$ cells, is already significantly compromised (1). The ability of the immune system to restore itself is critical and obviously has far-reaching implications for immune health, which includes the successful control of infection, as well as effective tissue homeostasis and repair. A dysfunctional immune system may also influence the patient's general health, contributing to the increased incidence of cardiovascular disease, neurocognitive dysfunction, and secondary cancer (2).

Longitudinal studies have determined that immune cell populations have differential rates of recovery after chemotherapy treatment, with innate (e.g., NK cells, monocytes) populations generally recovering to pretreatment numbers more rapidly than adaptive (e.g., T cells, B cells) populations. These studies demonstrate that the recovery of B cells occurs within 6-12 months, even surpassing pretreatment levels during this time (3). T cell recovery varied by subset; $C D 8^{+} \mathrm{T}$ cells appear to recover within 6 months, whereas $\mathrm{CD}^{+} \mathrm{T}$ cells, particularly the naive population, remain reduced at least out to 12 months. The relatively rapid increase in $\mathrm{CD}^{+} \mathrm{T}$ cells was attributed to the expansion of antigen-driven $\mathrm{CD} 28^{-}$effector cells in lymphopenic environments (4-6). However, the rebuilding of the adaptive immune system is a multifaceted process, as not only the size (i.e., quantity), but also the complexity and diversity (i.e., quality) of the system needs to be restored. Whether the overall quality of the adaptive immune system is fully recovered during this time period is unclear. 
Assessment of successful regeneration has to consider the numerical, compositional, and functional dimensions that signify a competent immune system. Indeed, one hallmark of the naive $\mathrm{T}$ and $\mathrm{B}$ cell compartments is their enormous diversity of antigen-specific receptors that enables the specific recognition of virtually all possible antigenic peptides. These naive cells also need to have stem-like properties including a high proliferative potential so that infrequent specificities can expand to large clonal sizes that can control pathogens. Moreover, they need to possess the pluripotency to differentiate into specific effector lineages under the appropriate environmental cues. Whether naive repertoire diversity can be maintained and/or rebuilt in adults after immune-ablation is currently unknown. Another unique feature of adaptive immunity is that the cumulative antigen experience over lifetime is stored as memory cells that are clonally expanded. Memory T cells (Tem) are poised for effector function to allow for rapid responses, while memory B cells can undergo progressive affinity maturation to improve antibody binding. This memory function is a pillar of immune competence and is successfully taken advantage of in vaccinations. To what extent the memory $\mathrm{T}$ and $\mathrm{B}$ cell compartments retain their antigen specificities after chemotherapy is also unclear but has obvious clinical implications for revaccination strategies.

The mechanisms that are involved in lymphocyte generation and that therefore ultimately determine the rebuilt repertoire are different for naive and memory B and T cells and are highly dependent on age and the degree of lymphopenia. The rules of naive B cell regeneration in chemotherapy-treated breast cancer patients is likely similar to early life, with the difference being that stem cells in middle-aged to older adults are less competent to differentiate into B cells $(7,8)$. The setting is different for T cells; generation of potentially novel $\mathrm{T}$ cells is strictly dependent on thymic activity. By the age of 50 years or older, which is the age of many breast cancer patients undergoing chemotherapy, the thymus has mostly involuted, leaving individuals with less than $1 \%$ of the output of young adults (9). Under steady state conditions, the vast majority of $\mathrm{T}$ cell generation in adults, therefore, comes from homeostatic proliferation. Under conditions of lymphopenia, the thymus may regain some activity. Indeed, using imaging as well as quantification of $\mathrm{T}$ cell receptor excision circles, Hakim et al. have estimated a thymus volume increase in 50\% of BM transplant recipients between the age of 40 and 50 years and $10 \%$ in patients older than 50 years (10). However, lymphopenia also clearly induces homeostatic proliferation, with an increase in cell turnover rates. Experiments in the mouse have shown that the functional outcome of lymphopenic proliferation is different from that of steady-state maintenance, with naive cells entering differentiation and expressing a distinct phenotype $(11,12)$. This phenotype is similar to described virtual Tem (i.e., cells that have never encountered foreign antigen but still develop memory features with age; refs. 13-15). Thus, severe lymphopenia, such as that caused by chemotherapy, likely changes not only the numbers, but potentially the phenotype of classically naive $\mathrm{T}$ cells.

Unlike the naive compartment, Tem are generated in a number of ways: differentiation of naive $\mathrm{T}$ cells, cytokine-driven homeostatic proliferation of surviving Tem, and antigen-driven proliferation. Expansion of the $\mathrm{CD} 28^{-}$population of $\mathrm{CD}^{+} \mathrm{T}$ cells, as seen early in breast cancer patients, is commonly driven by antigen-specific mechanisms, such as latent CMV infection (16). During aging, there is a similar expansion of CD28- cells that persists over the course of a lifetime (17). Moreover, Tem are a highly heterogenous population, containing numerous subsets of cells with unique phenotypic and functional properties, driven both by antigen and environment signals. Changes in these memory subsets, including their relative distributions and phenotypic/functional profiles, contribute to many immune dysfunctions.

Newly developed analytic approaches allow a significantly improved assessment on the complexity of the adaptive immune system. Multidimensional mass cytometry of phenotypic markers with high-dimensional clustering provides better resolution of subset composition and allows for conclusions on the origin and functional state of a population. Next-generation sequencing of $\mathrm{T}$ cell receptor genes also is a more recent tool to quantify repertoire diversity, in particular when used in combination with statistical analysis of replicate samples, and can provide insight into $\mathrm{T}$ cell repopulation mechanisms. The current study examined adaptive immune cell populations, particularly in the $\mathrm{T}$ cell compartment, for their ability to recover — in quantity and quality — in middle-aged and older individuals after a lymphopenia-inducing event such as adjuvant chemotherapy. Here, we describe unique regeneration patterns of different immune cell subsets. $\mathrm{CD}^{+} \mathrm{T}$ cell composition almost completely resolves after 1 year. Memory B cells are transiently depleted for the first year after treatment but recover within 3-5 years. Conversely, the $\mathrm{CD} 4^{+} \mathrm{T}$ cell compartment is severely affected both in quantity and quality for more than 5 years, with a significant diminution of naive cells and a long-lasting shift in $\mathrm{CD} 4^{+}$Tem toward more inflammatory CD $161^{+}$cells. In spite of these changes, $\mathrm{T}$ cell receptor repertoire diversity in naive and $\mathrm{CD} 4^{+} \mathrm{Tem}$ is maintained, implying unbiased and 
non-antigen-driven regeneration mechanisms. Together, these data reveal the complexity of the adaptive immune system, in which individual subsets display unique regenerative capacities. Moreover, these data highlight the potential need for new vaccination strategies to restore full immune competence in adjuvant chemotherapy-treated breast cancer patients.

\section{Results}

Composition of peripheral blood mononuclear cells in breast cancer patients later than 1 year after adjuvant chemotherapy. Using multidimensional mass cytometry, we compared the immune cell composition of peripheral blood mononuclear cells (PBMCs) from breast cancer patients who were 1 to 5 years beyond chemotherapy treatment (Supplemental Table 1; supplemental material available online with this article; https://doi.org/10.1172/jci.insight.134569DS1) with that of age- and sex-matched controls who never had cancer nor received chemotherapy. Longitudinal monitoring of differential white blood cell counts showed a decline of about $50 \%$ in peripheral total lymphocyte numbers upon chemotherapy treatment in our patient cohort - a decline that was recovered by 1 year after chemotherapy (Supplemental Figure 1). For the initial analysis, we used classical hand-gating of mass cytometry data to determine relative frequencies of common immune cell subsets. The gating strategy for each immune cell population is provided in Supplemental Figure 2A. This analysis revealed slightly increased frequencies of B cells, although not statistically significant, with a trending decline in frequencies of plasmacytoid DCs (pDCs) and monocytes in patients compared with controls (Figure 1A and Supplemental Figure 2, B and $\mathrm{C}$ ). There were no differences in frequencies of myeloid DCs (mDCs), NK cells, $\gamma \delta \mathrm{T}$ cells, NK $\mathrm{T}$ cells, $\mathrm{CD}^{+} \mathrm{T}$ cells, or $\mathrm{CD} 4^{+} \mathrm{T}$ cells. Thus, these initial results indicate that the immune system of breast cancer patients mostly regains a composition of global immune cell subsets similar to that of healthy controls within 1 year after chemotherapy treatment.

However, the analysis of mass cytometry by hand-gating disregards the information that is included in the high dimensionality and complexity of immune cell subsets. Thus, to increase the resolution of the analysis, we used a high-dimensional, unbiased clustering program, "Statistical Scaffold," that simultaneously allows for statistical comparisons of populations (or "cluster") frequencies across experimental groups (18). In agreement with the finding from hand-gating, we found that cell clusters linked with pDCs and monocytes had decreased frequencies in patients (Figure 1B). Moreover, we found that multiple B cell clusters increased in patients. Again, no differences in $\mathrm{mDC}$, NK cell, $\gamma \delta \mathrm{T}$ cell, and NK T cell frequencies were detected. Most notably, although we found no difference in total $\mathrm{CD}^{+} \mathrm{T}$ cell frequencies in Figure 1A, there were major cluster differences between patients and controls within the $\mathrm{CD}^{+} \mathrm{T}$ cell compartment, both with clusters increasing and decreasing in frequencies (Figure 1B). Major cluster differences were not observed within the $\mathrm{CD} 8^{+} \mathrm{T}$ cell compartment, where most clusters had similar frequencies between patients and controls. Together, these data suggest that alterations in $\mathrm{T}$ cell composition in patients are $\mathrm{CD} 4$, but not CD8, T cell selective.

Alterations in $C D 4^{+}$and $C D 8^{+} T$ cell subsets in chemotherapy-treated patients. Compared with $\mathrm{CD}^{+}$ $\mathrm{T}$ cells, $\mathrm{CD}^{+} \mathrm{T}$ cell homeostasis - and therefore regeneration - is usually more vulnerable to stressors such as latent viral infection or aging $(17,19)$. It was therefore surprising to find that chemotherapy-induced differences in $\mathrm{T}$ cell composition were mainly in the $\mathrm{CD} 4^{+} \mathrm{T}$ cell and not the $\mathrm{CD} 8^{+} \mathrm{T}$ cell compartment. To get a better understanding of the differences in the regeneration of $\mathrm{T}$ cell compartments of patients, we performed high-resolution Statistical Scaffold on $\mathrm{CD}^{+}$or $\mathrm{CD} 8^{+} \mathrm{T}$ cell populations, using major phenotypic (e.g., naive, central memory, effector memory) and functional (e.g., $\mathrm{T}$ follicular helper cell, Treg, Th17) $\mathrm{T}$ cell subsets as predefined landmark nodes. The gating strategy for each of these $\mathrm{T}$ cell subsets, determined by surface marker expression $(20,21)$, is provided in Supplemental Figure 3. Hand-gating detected multiple CD4 subsets with differential frequencies between patients and controls (Supplemental Table 2). Moreover, the CD4 scaffold analysis showed striking and consistent differences in the $\mathrm{CD}^{+} \mathrm{T}$ cell compartment between patients and controls (Figure 2A). As anticipated, multiple clusters linked with the naive landmark nodes were decreased in patients, whereas multiple memory clusters were increased. However, we additionally found significant expansion of clusters close to more proinflammatory memory phenotypes (Th1, Th17) - a phenomenon that was observed across multiple patients. Clusters linked with the terminal differentiated effector memory $\left(\mathrm{T}_{\mathrm{EMRA}}\right)$ node, which mainly includes $\mathrm{T}$ cells specific to latent viruses, did not show differences between patients and controls. 


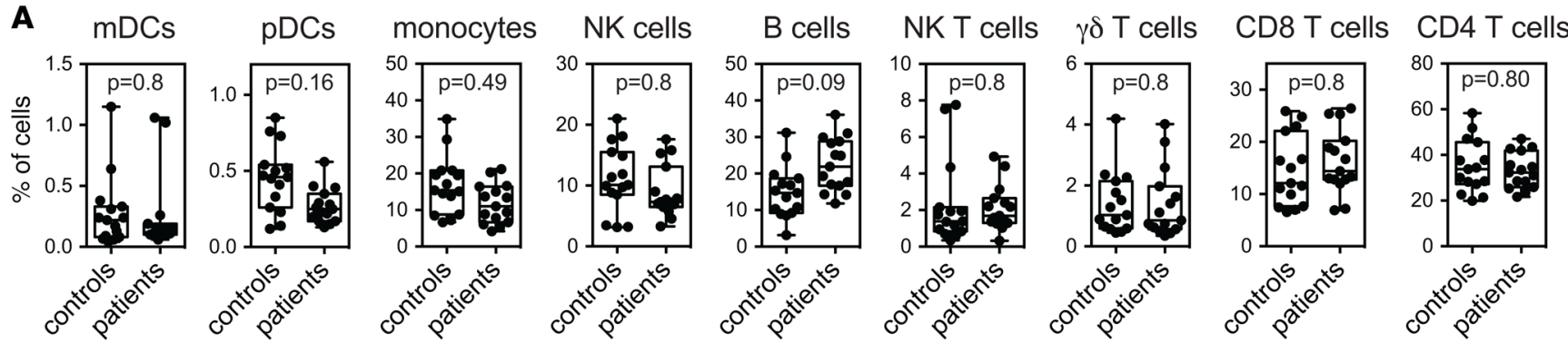

\section{B}

Control

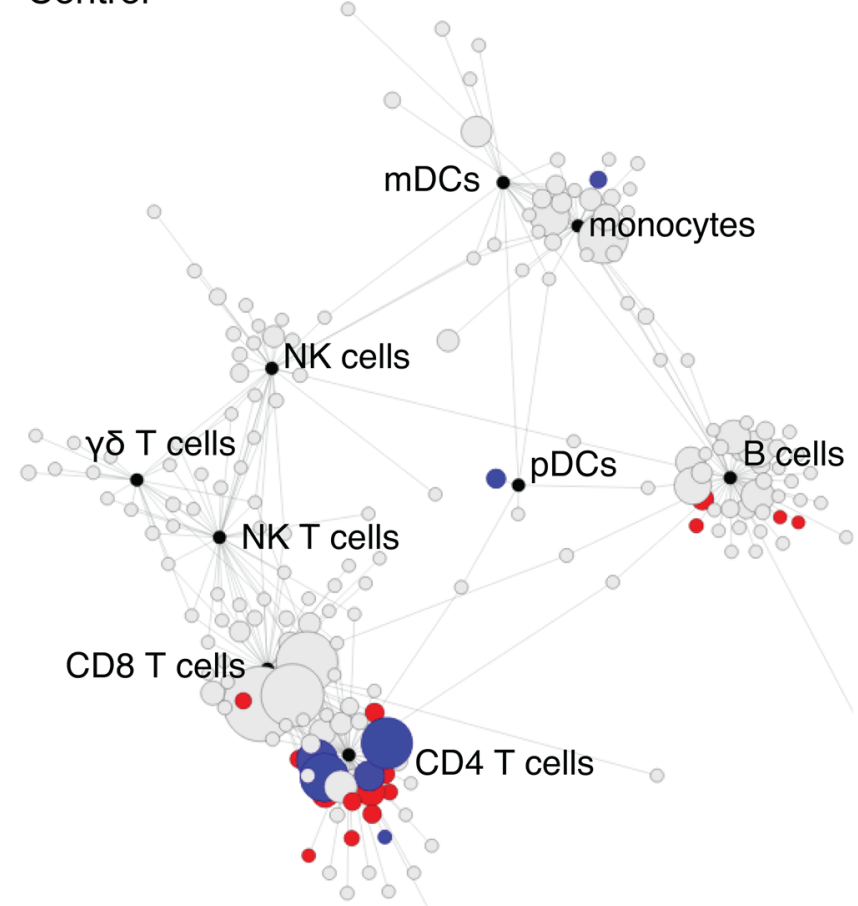

Patient

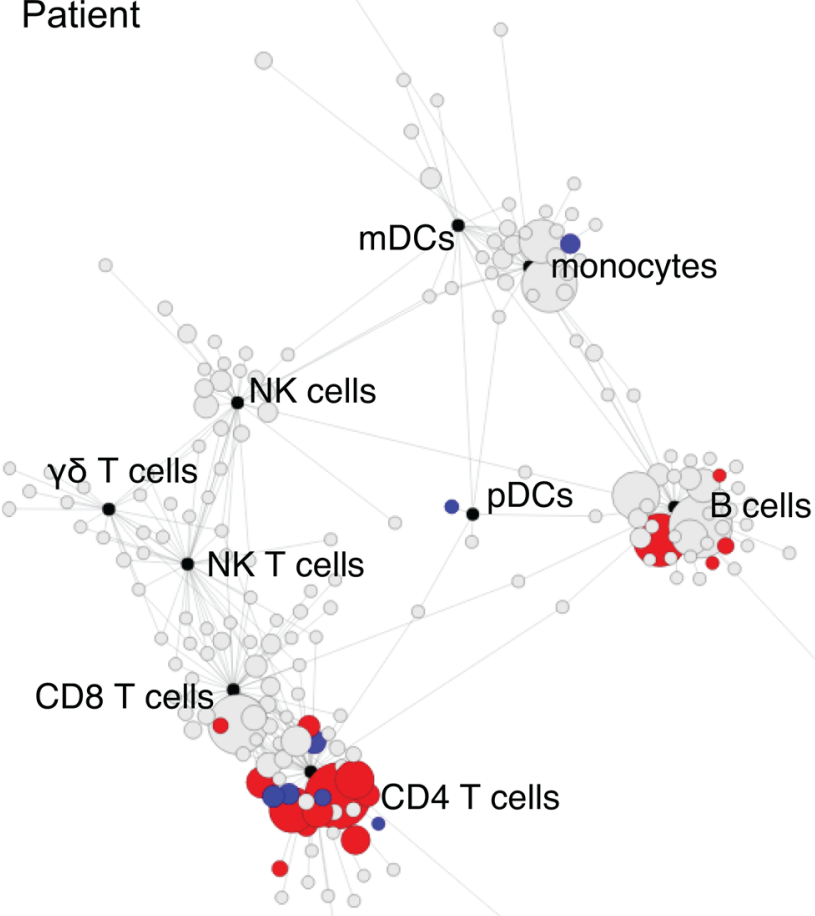

Colored by q-value: red = increased in patients blue = decreased in patients, gray = No change black = landmark node

Figure 1. Effect of adjuvant chemotherapy on circulating immune cell subsets in breast cancer patients. (A) Frequencies of immune cell populations in peripheral blood from healthy controls $(n=15)$ and chemotherapy treated breast cancer patients $(n=15)$, as determined by hand-gating of data from 33 parameter mass cytometry. $P$ values were determined using Mann-Whitney $U$ tests with Hochberg multiple comparisons. (B) Scaffold plots of live, single cells from 1 healthy control (left) and 1 patient (right) using 200 cluster input. Red indicates that a cluster is statistically significantly higher in patients, and blue is statistically lower in patients, while gray is no difference, based on $Q$ values calculated in Statistical Scaffold analysis comparing all 15 patients to 15 age-matched healthy controls. Black nodes are landmark nodes, which indicate where a specific cell population lies in relationship to the cluster. The size of the cluster is relative to its proportion within total cells within a sample, where larger clusters contain a higher number of cells. Distance is relative to how similar clusters are to landmark nodes.

In contrast to the $\mathrm{CD} 4$ analysis, the $\mathrm{CD} 8^{+} \mathrm{T}$ cell analysis revealed very few cluster differences between patients and controls (Figure 2B). Again, no clusters linked with $\mathrm{T}_{\text {EMRA }}$ nodes were different, implying that repopulation is not biased for $\mathrm{CD} 8^{+} \mathrm{T}$ cells specific for latent viruses. Overall, the $\mathrm{CD} 4^{+} \mathrm{T}$ cell compartment exhibited significantly more differentially expressed clusters than the $\mathrm{CD} 8^{+} \mathrm{T}$ cell compartment; the $\mathrm{CD} 4^{+}$ $\mathrm{T}$ cell compartment had $50 \%$ of clusters, whereas the CD8 compartment only had $8 \%$ clusters differentially expressed between patients and controls (Figure 2C). Additionally, many more subsets were affected in the $\mathrm{CD}^{+} \mathrm{T}$ cells compared with $\mathrm{CD} 8^{+} \mathrm{T}$ cells (Figure 2D). Indeed, the increased memory clusters in the $\mathrm{CD} 4^{+}$ 
A

CD4 T cells

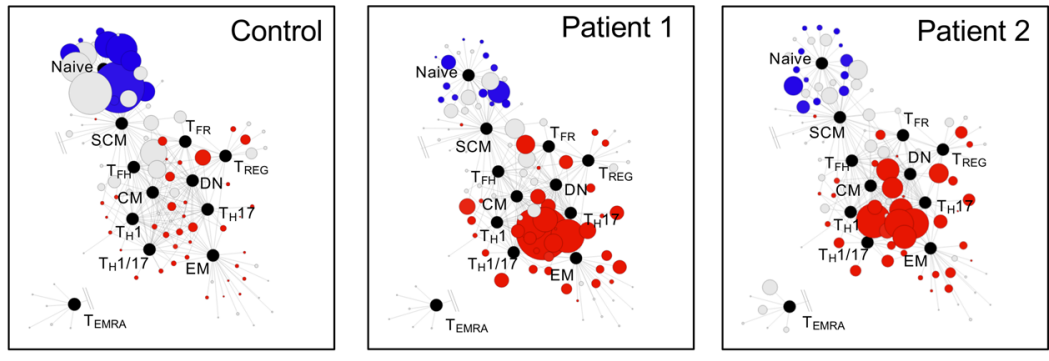

Colored by q-value: red $=$ increased in patients, blue $=$ decreased in patients, gray $=$ No change, black = landmark nod

B CD8 T cells
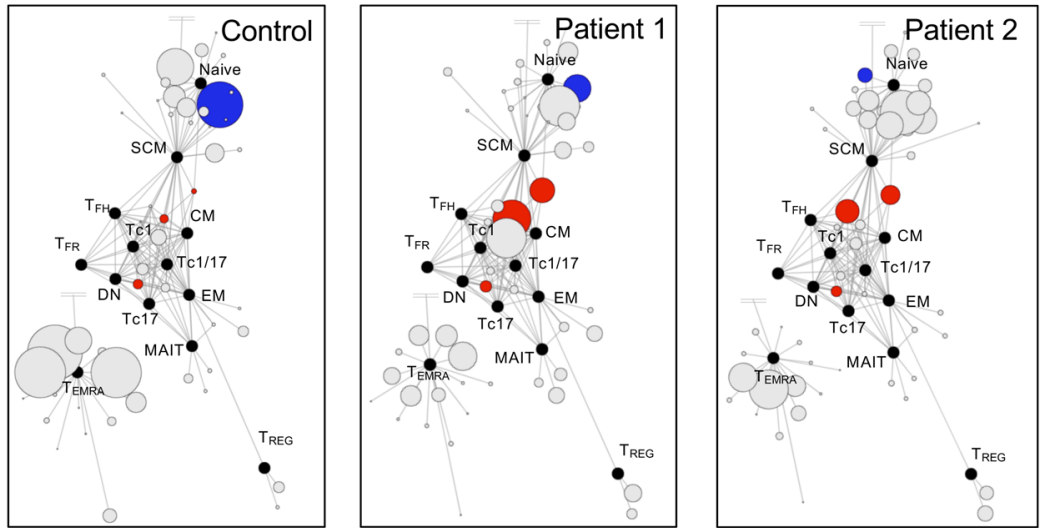

Colored by q-value: red = increased in patients, blue = decreased in patients, gray = No change, black = landmark node
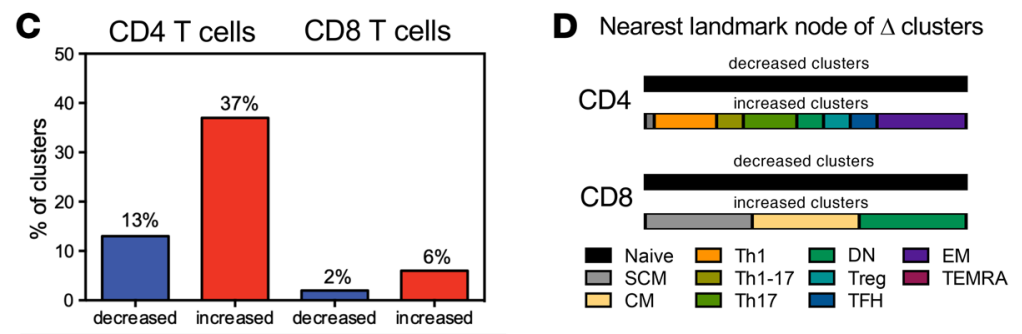

Figure 2. Major alterations in the $\mathrm{CD4}^{+} \mathrm{T}$ cell compartment with minor $\mathrm{CD8}^{+} \mathrm{T}$ cell changes in patients postchemotherapy. (A) Representative scaffold plots for $C D 4^{+} T$ cells from 15 patients and 15 healthy controls using 100 clusters. Cluster size is proportional to cell frequencies in that donor. (B) Representative scaffold plots for CD8 $8^{+}$cells from 15 patients and 15 healthy controls using 50 clusters. Cluster size is proportional to cell frequencies in that donor. (C) The percentage of differential clusters between patients and controls in $\mathrm{CD} 4^{+}$and $\mathrm{CD} 8^{+} \mathrm{T}$ cell scaffold analyses. (D) The subset composition of differential clusters in $\mathrm{CD} 4^{+}$and $\mathrm{CD} 8^{+} \mathrm{T}$ cells, based on the highest predicted nearest landmark node.

T cell compartment where spread across the phenotypic and functional subsets, whereas only 3 memory compartment (e.g., stem-like memory, central memory, and CXCR3-CCR6- double negative [DN] cells) in $\mathrm{CD}^{+} \mathrm{T}$ cells were linked with differential cluster expression. Thus, these data indicate that, unlike the $\mathrm{CD} 8^{+} \mathrm{T}$ cell compartment, the $\mathrm{CD} 4^{+} \mathrm{T}$ cell compartment indeed has major population reorganization after chemotherapy that includes reductions in naive cells and expansions in multiple memory subsets.

Naive $T$ cell repopulation and maintenance in chemotherapy-treated patients. Naive regeneration can occur via multiple mechanisms: thymic production, homeostatic proliferation, and - recently discovered — infiltration of the naive pool with naive-like memory cells. Thus, we sought to better understand naive $\mathrm{CD} 4^{+}$and $\mathrm{CD} 8^{+} \mathrm{T}$ cell regeneration by elucidating naive population composition. Using classical CCR $7{ }^{+} \mathrm{CD} 45 \mathrm{RA}{ }^{+}$ hand-gating strategy for defining the naive population, we found that naive $\mathrm{CD} 4^{+} \mathrm{T}$ cells were significantly lower in patients than in controls (Figure 3, A, C, and D), consistent with the high-dimensional analysis. Also, there was no difference in naive $\mathrm{CD} 8^{+} \mathrm{T}$ cells between the 2 groups, demonstrating that the naive CD8 compartment was able to fully repopulate within a year after chemotherapy, whereas the CD4 compartment was not. Increased thymic output was observed in both naive CD4 and CD8 subsets, with T cell 
A
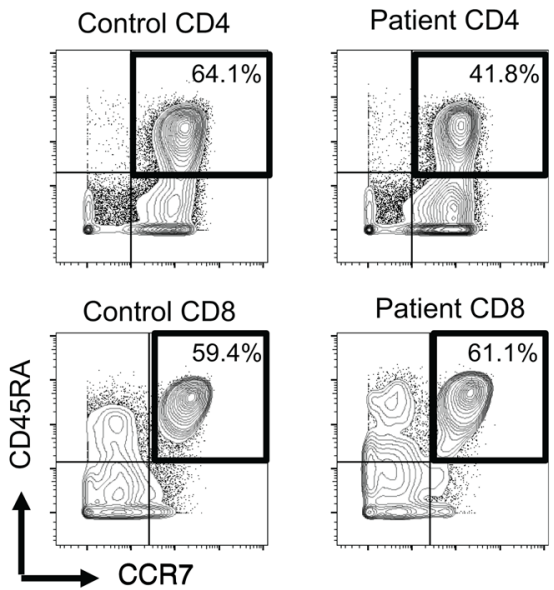

B
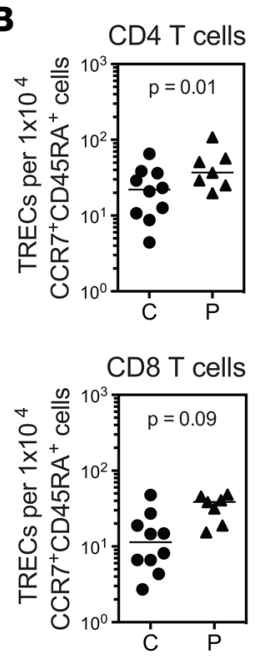
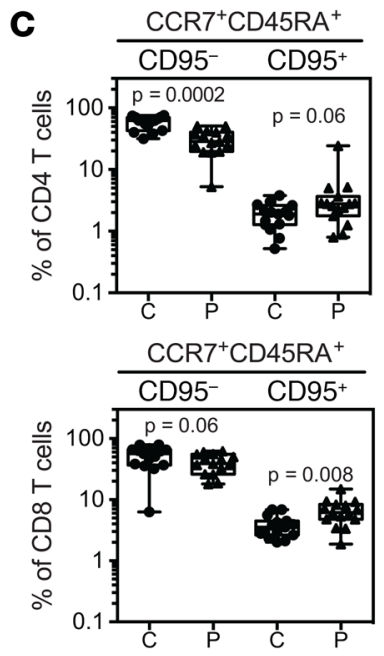

D
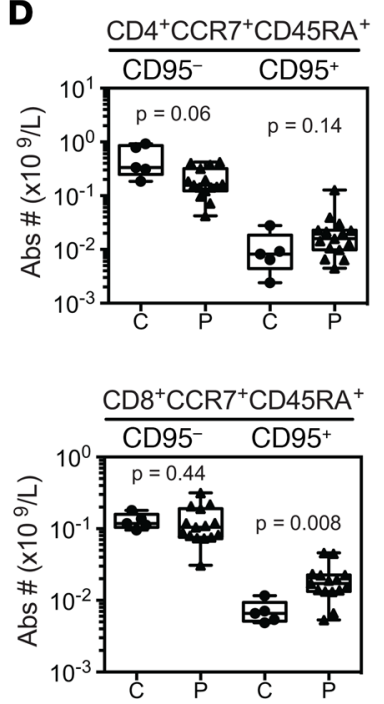

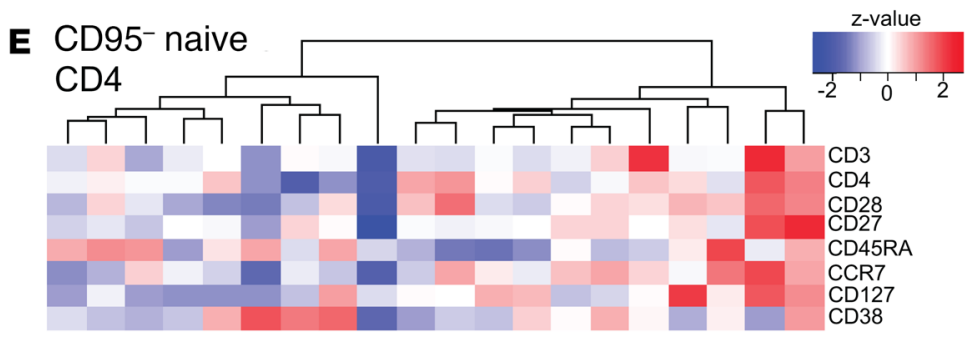

C5 C3 C4 P2 P5 P3 P7 P9 P1 C1 C2 C9 P4 P8 C8 C10 C7 C6 P10 P6

$\mathbf{F}$

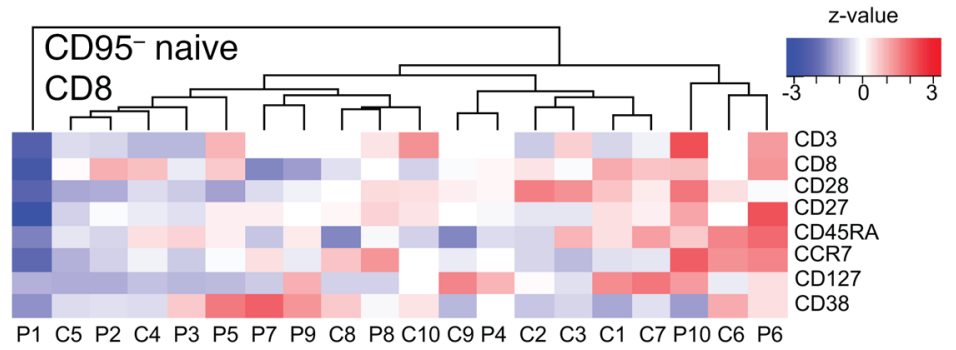

Figure 3. Reduced naive $C D 4^{+} \mathbf{T}$ cells and expanded $C D 95^{+}$naive $\mathbf{C D 8}{ }^{+} \mathbf{T}$ cells in patients. (A) Representative flow plots of $\mathrm{CD4}{ }^{+}$and $\mathrm{CD} 8^{+}$naive (CCR7 ${ }^{+}$CD45RA ${ }^{+}$) T cell frequencies in patients and controls. (B) Number of T cell excision circles (TRECs) present in CD4 (upper) and CD8 ${ }^{+}$(lower) naive

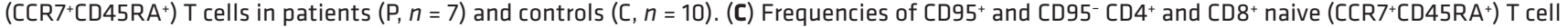
frequencies within total CD4 and CD8 compartments of patients $(n=15)$ and controls $(n=15)$. (D) Absolute cell numbers of CD95 and CD95- CD4 ${ }^{+}$and CD8 $^{+}$naive $\left(C C R 7^{+}\right.$CD45RA $\left.^{+}\right)$T cells in patients $(n=15)$ and controls $(n=5)$. ( $E$ and $\left.\mathbf{F}\right)$ Heatmap of median surface marker expression intensity on CD95 $\mathrm{CD}^{+}(\mathbf{E})$ and $\mathrm{CD}^{+}(\mathbf{F})$ naive $\left(C C R 7^{+} \mathrm{CD}_{\left.45 \mathrm{RA}^{+}\right)}\right.$T cells from patients $(n=10)$ and controls $(n=10)$ using unsupervised hierarchical clustering. $P$ values were determined by Mann-Whitney $U$ tests with Hochberg multiple comparisons.

receptor excision circles (TRECs) higher in patients than controls for both $\mathrm{CD}^{+}$and $\mathrm{CD} 8^{+}$naive $\mathrm{T}$ cell subsets, from $11.4-38.6$ cells per $1 \times 10^{4}$ naive $\mathrm{CD} 4^{+} \mathrm{T}$ cells and from $22-37$ cells per $1 \times 10^{4}$ naive $\mathrm{CD} 8^{+}$ $\mathrm{T}$ cells (Figure $3 \mathrm{~B}$ ). However, naive $\mathrm{CD} 4^{+} \mathrm{T}$ cells showed a greater increase in thymic output compared with naive $\mathrm{CD} 8^{+} \mathrm{T}$ cells (3.5-fold increase vs. 1.7-fold increase), suggesting that biased $\mathrm{T}$ cell output from the thymus is not the driving factor in incomplete naive CD4 regeneration.

The naive compartment can also be repopulated by a subpopulation of memory cells that have reacquired a naive phenotype $(22,23)$. These cells, often termed stem-like memory cells, are commonly delineated from naive cells by the expression of CD95. Thus, we further investigated the difference in $\mathrm{CD}^{-} 5^{-}$and $\mathrm{CD}^{2} 5^{+}$naive cells. Within $\mathrm{CD}^{+} \mathrm{T}$ cells, there was a significant decrease in the percent of $\mathrm{CD}^{-5} 5^{-}$naive cells in patients compared with controls ( $28.5 \%$ vs. $62.4 \%$ median, respectively) (Figure $3 \mathrm{C}$ ). Although $\mathrm{CD} 95^{+}$naive $\mathrm{CD} 4^{+} \mathrm{T}$ cells trended higher, they were not statically different. Absolute numbers of $\mathrm{CD} 95^{-}$naive $\mathrm{CD} 4^{+} \mathrm{T}$ cells were also reduced in patients, with no differences in $\mathrm{CD}^{2} 5^{+}$numbers (Figure 3D). Conversely, $\mathrm{CD} 95^{-}$naive $\mathrm{CD} 8^{+} \mathrm{T}$ cells 
were similar in frequency and number to that of controls, whereas $\mathrm{CD} 95^{+}$naive $\mathrm{CD} 8^{+} \mathrm{T}$ cells were significantly increased in patients (Figure 3, C and D). The interpretation of these data are 3-fold: (a) naive $\mathrm{CD}^{+} \mathrm{T}$ cell recover normally, whereas $\mathrm{CD} 4^{+} \mathrm{T}$ cells do not; (b) the limited regeneration of the naive CD4 compartment is not due to biases in thymic output; and (c) naive $\mathrm{CD} 8^{+}$, but not $\mathrm{CD}^{+}$, $\mathrm{T}$ cells have a significant expansion of naive-like memory cells that contributes to population regeneration.

The CD95 marker may not identify all Tem that reacquire a naive phenotype (24). To explore whether the composition of $\mathrm{CD}^{-} 5^{-}$naive $\mathrm{T}$ cells in patients is different from that of controls, we compared the median expression of multiple surface markers on these $\mathrm{CD} 4^{+}$and $\mathrm{CD} 8^{+} \mathrm{T}$ cell subsets between patients and controls using hierarchical clustering. Although there was high heterogeneity of surface marker expression between individuals, there were similar expression patterns in patients and controls and no specific separation of clusters with patients and controls in $\mathrm{CD} 95^{-}$naive $\mathrm{CD}^{+} \mathrm{T}$ cells (Figure $3 \mathrm{E}$ ). Similar profiles and expression levels of these markers were also observed in $\mathrm{CD} 95^{-}$naive $\mathrm{CD} 8^{+} \mathrm{T}$ cells (Figure $3 \mathrm{~F}$ ). Thus, naive population regeneration does not appear to be influenced by $\mathrm{CD} 95^{-}$naive-like memory cells.

Memory $T$ cell repopulation and maintenance in chemotherapy-treated patients. High-dimensional analysis indicated major changes within the $\mathrm{CD}^{+}$memory $\mathrm{T}$ cell compartment — highlighted by significant increases in clusters phenotypically around central memory cells and functionally close to Th17, Th1/17, and Th1 cells (Figure 2A). Therefore, we further analyzed the phenotypic and functional subsets of CD4 ${ }^{+}$ Tem from patients and controls. Overall, the frequency of central memory $\left(\mathrm{CCR} 7^{+} \mathrm{CD} 45 \mathrm{RA}{ }^{-}\right) \mathrm{CD}^{+}$ $\mathrm{T}$ cells was significantly increased in patients compared with controls $(54.3 \%$ vs. $31.6 \%$, respectively) (Figure 4A). Absolute numbers exhibited similar increases (data not shown). We next determined the composition of functional subsets (i.e., Th1, Th17, and Th1/17) within the central memory compartment. Subset definitions were deduced from the surface expression patterns of CXCR3 and CCR6 (Figure 4B and ref. 20). As suggested by the $\mathrm{CD}^{+} \mathrm{T}$ cell scaffold analysis (Figure 2), Th17 and Th1/17 subsets were significantly increased in frequency and by absolute counts in patients (Figure 4, C and D). The Th1 subset trended higher by absolute counts but not by relative frequency. The CXCR3-CCR6 ${ }^{-}$DN subset decreased in frequency but not in absolute number. Together, these data suggest that Th17-like cells are preferentially expanded in patients. To further support this interpretation, we determined the expression of CD161, a known (and independent) marker of Th17 cells (25), within the CD4 central memory population. Consistently, $\mathrm{CD} 161^{+}$central memory cells were increased, both by frequency and absolute counts, in patients compared with controls (Figure 4E).

In patients, the central memory CD4 compartment displayed a Th17-like skewing of its composition; thus, we determined whether central memory $\mathrm{CD}^{+} \mathrm{T}$ cells display similar features. The composition of the central memory $\mathrm{CD}^{+} \mathrm{T}$ cells exhibited a slight but not significant increase in frequencies of the $\mathrm{T}_{\mathrm{C}} 17$ subset, but no change for $\mathrm{Tc} 1 / 17$, $\mathrm{Tc} 1$, or DN cells (Figure $4 \mathrm{~F}$ ). However, unlike $\mathrm{CD}^{+} \mathrm{T}$ cells, no increase in CD161 expression was found within the central memory CD8 compartment in patients (Figure 4G), both for the CD161 ${ }^{\text {hi }}$ population (which are mucosal-associated invariant $\mathrm{T}$ cells or "MAIT" cells), as well as for the CD161 $1^{\mathrm{lo} /+}$ population. Therefore, the CD $4^{+} \mathrm{T}$ cell, but not the CD $8^{+}$ $\mathrm{T}$ cell, compartment in patients demonstrates major skewing of the central memory functional phenotypes, highlighted by an expansion of CD161+ Th17-like memory cells.

Effects of adjuvant chemotherapy on $T$ cell receptor diversity. With thymic activity insufficient to completely restore the naive T cell compartment in older adults, loss of entire T cell clones (i.e., clonotypes) caused by chemotherapy would not be recovered. Moreover, T cell replenishment by homeostatic proliferation may favor the expansion of specific clonotypes. Chemotherapy may therefore result in significant TCR repertoire contraction, similar to that seen with aging. To estimate TCR diversity, we sorted naive $\left(\mathrm{CD} 45 \mathrm{RA}^{+} \mathrm{CCR} 7^{+} \mathrm{CD} 28^{+}\right)$and $\mathrm{CD} 4^{+}$and $\mathrm{CD} 8^{+}$Tem populations from 7 patients and 7 age-matched healthy controls and performed next-generation $T R B$ sequencing. As previously described, 5 replicates of each population were sequenced for each individual, allowing for robust estimates of TCR richness (i.e., the number of unique TCR sequences) and clonality (i.e., the extent of clonal size variation) (26). In spite of the significant reductions in naive $\mathrm{CD}^{+} \mathrm{T}$ cell numbers in patients (Figure 3), TCR richness of the naive CD4 compartments of patients and controls were not significantly different (Figure 5A). Surprisingly, even with an increased frequency of $\mathrm{CD}^{2} 5^{+}$cells within the $\mathrm{CD} 45 \mathrm{RA}{ }^{+} \mathrm{CCR} 7^{+} \mathrm{CD} 8^{+} \mathrm{T}$ cell population of patients (Figure 3 ) that, if deriving from memory cells, would be less diverse, TCR richness in the naive $\mathrm{CD}^{+} \mathrm{T}$ cells trended higher in patients compared with controls. Likewise, both $\mathrm{CD} 4^{+}$and $\mathrm{CD} 8^{+} \mathrm{Tem}$ showed similar TCR richness between patients and controls (Figure 5B), consistent with the interpretation 


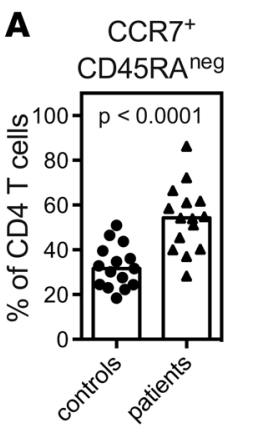

$\mathbf{B}$
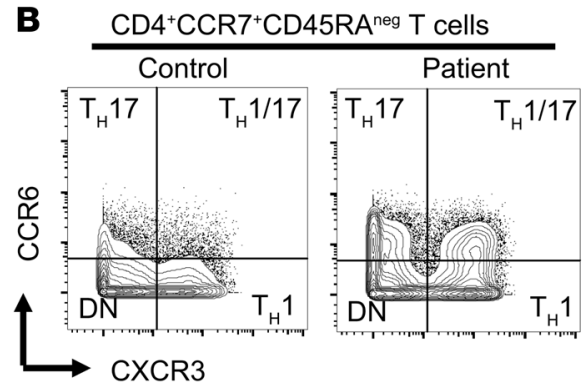

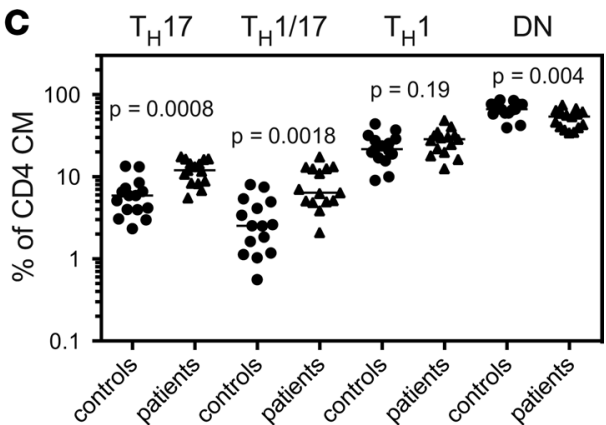

E
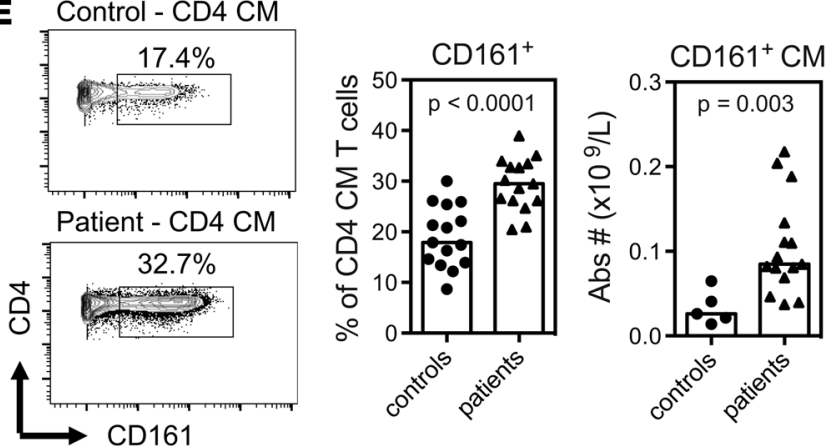

G
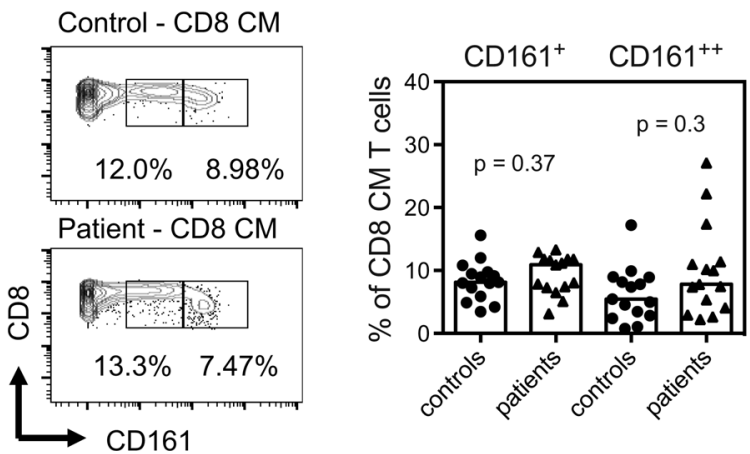

Figure 4. Expansion of Th17-like central memory populations in the $\mathrm{CD4}^{+} \mathrm{T}$ cell compartment in patients. (A) Frequencies of central memory (CCR7 ${ }^{+}$CD45RA $)$CD4 $^{+}$T cells within the CD4 ${ }^{+}$T cell compartment of patients $(n=15)$ and controls $(n=15)$. (B) Representative flow plots of CXCR3 and CCR6 on central memory CD4+ T cells from patients and controls, indicating the associated functional subset definition; Th1, Th17, Th1/17, and CXCR3-CCR6- (DN) cells. (C) Frequencies of functional subsets in the central memory CD4 ${ }^{+}$T cell compartment of patients $(n=15)$ and controls $(n=15)$. (D) Absolute numbers of Th1, Th17, Th1/17, and DN central memory CD4+T cells in patients $(n=15)$ and controls $(n=5)$. $(E)$ Frequencies and absolute number of CD161+ central memory CD4+ T cells in patients and controls. (F) Frequencies of functional subsets (Tc1, Tc17, Tc1/17, and DN) in the central memory CD8 ${ }^{+}$T cell compartment of patients $(n=15)$ and controls $(n=15)$. (C) Frequencies and absolute number of $C D 161^{+}$and $C D 161^{++}$ central memory $C D 8^{+}$T cells in patients and controls. $P$ values were determined by Mann-Whitney $U$ tests with Hochberg multiple comparisons.

that chemotherapy does not induce complete elimination of naive and memory clonotypes. Partial thymic rejuvenation, seen by increased TRECs in patients (Figure 3B), may account for the increased richness in patients' naive CD8 repertoire.

Similar to TCR richness, there was no significant differences in repertoire clonality between patients and controls in either the naive or memory compartments (Figure 5, C and D). These data indicate that repopulation through expansion of clonotypes was not uneven (Figure 5, C and D), unlike the expansions observed with chronic viral infection that are highly biased toward a small subset of clonotypes. Taken together, the similar richness and clonality of TCR repertoires between patients and controls reveals that adjuvant chemotherapy treatment does not compromise overall TCR repertoire diversity and that population regeneration is unlikely to be driven by the expansion of virus-specific clonotypes (e.g., CMV-specific T cells).

Naive $\mathrm{T}$ cell regeneration is complex, as homeostatic proliferation of naive $\mathrm{T}$ cells causes differentiation into virtual memory cells and long-term $\mathrm{CD}^{+}$Tem can reacquire a naive phenotype. To understand whether 
A
B

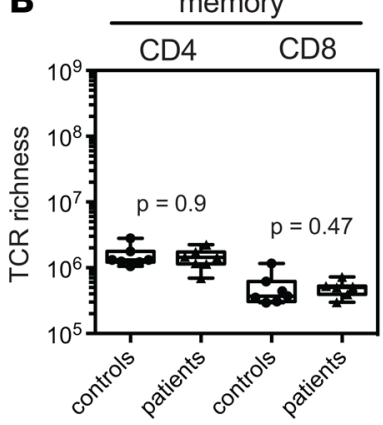

E
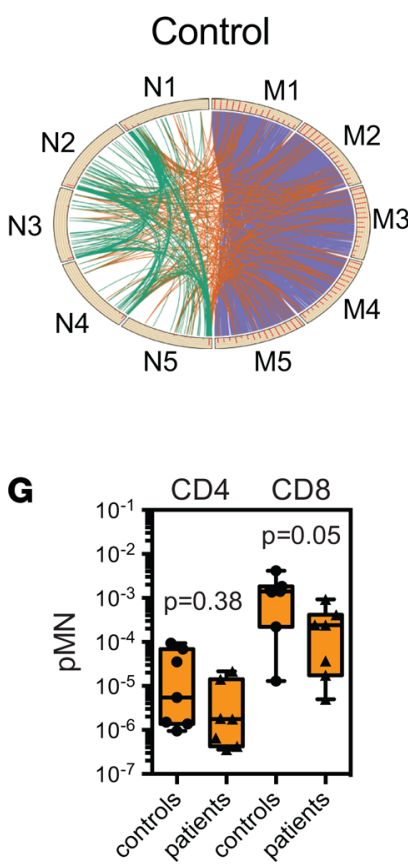

\section{CD4}
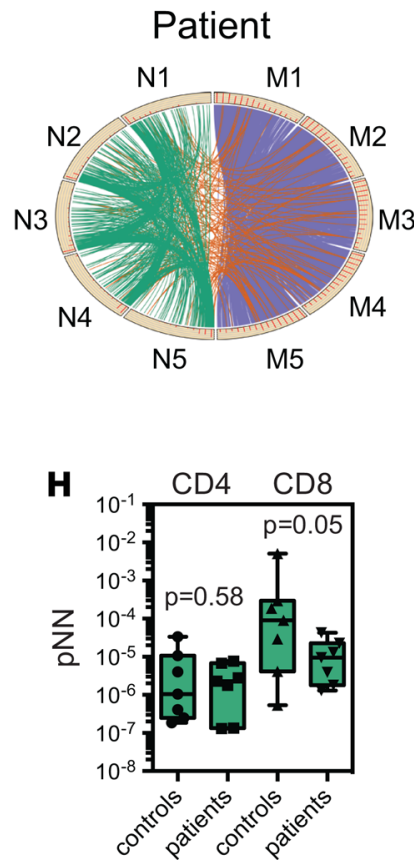
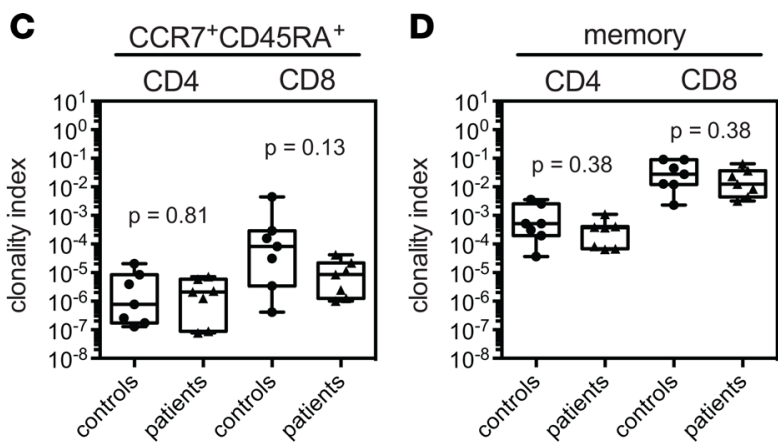

$\mathbf{F}$

CD8

Control

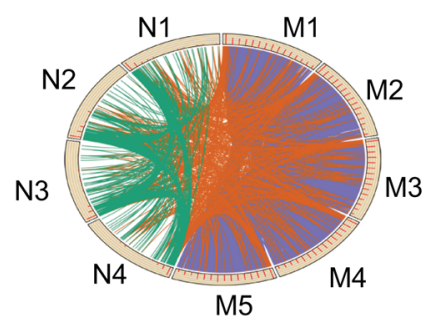

Patient

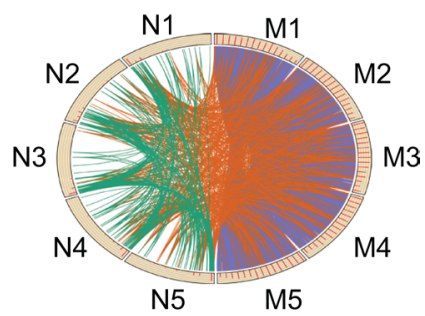

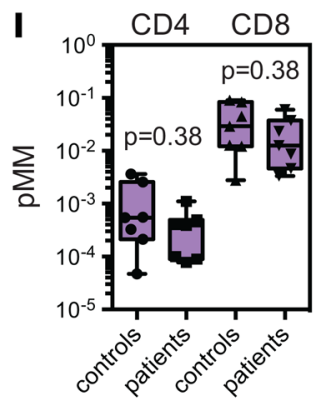

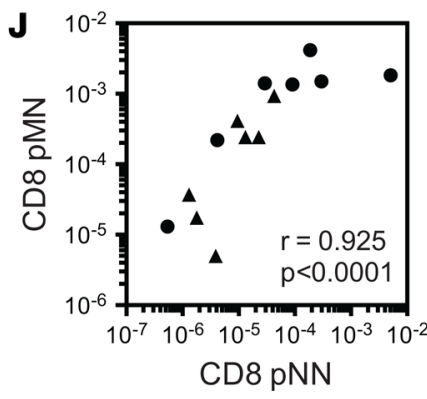

Figure 5. TCR repertoire diversity in naive and memory $T$ cell compartments after chemotherapy. TRB sequencing was performed on 7 chemotherapy-treated patients and 7 age-matched healthy controls. ( $A$ and $\mathbf{B})$ TCR richness of naive (CCR7 ${ }^{+}$CD45RA $)(\mathbf{A})$ and memory (B) CD4 $4^{+}$and CD8 $8^{+}$T cells in patients and controls. This parameter estimates the number of unique TRB sequences in a repertoire. (C and D) Clonality index of naive (CCR7 ${ }^{+}$CD45RA $\left.{ }^{+}\right)(\mathbf{C})$ and memory (D) $C D 4^{+}$and $C D 8^{+} T$ cells in patients and controls. This parameter estimates the amount of clonal expansion in a TCR repertoire. Both TCR richness and clonality index are based on TCR- $\beta$ V (BV), BD, and BJ segment usage and CDR3 amino acid sequence. $P$ values were determined by Mann-Whitney $U$ test. ( $E$ and $\mathbf{F}$ ) Representative Circos plots showing the overlap between the top 300 CDR3 amino acid sequences between naive and $\mathrm{CD} 4^{+}$Tem (E) and CD8+ $\mathrm{T}$ cells (F). Overlaps between naive-naive are green, memory-memory are purple, and naive-memory are orange. N1-N5 indicate replicates of naive and M1-M5 indicate replicates of memory cells from the same donor. (G-I) Quantification of TRB overlap between CDR3 nucleotide sequences between the naive and memory compartment (pMN) (G), in the naive compartment (pNN) (H), and in the memory compartment (pMM) (I) of patients and controls. $P$ values were determined by Mann-Whitney $U$ test. (J) Spearman's correlation between pNN and pMN in the $\mathrm{CD}^{+} \mathrm{T}$ cell compartment from patients (triangles) and controls (circles).

regeneration maintains or breaks the boundaries of phenotypically defined naive and Tem, we initially determined the overlap between the top 300 TCR clones in the naive and memory compartments (Figure 5, E and F). As seen from the Circos plots, overlap between the naive and memory compartments was (a) less frequent for $\mathrm{CD}^{+} \mathrm{T}$ cells compared with $\mathrm{CD} 8^{+} \mathrm{T}$ cells and (b) not significantly different between patients and controls. As expected, the memory replicates had high overlap with each other, indicative of larger clonal sizes and consistent with lesser TCR richness. Overlaps between naive and memory cells within the $\mathrm{CD}^{+} \mathrm{T}$ cell compartment were more frequent in both healthy controls and patients compared with $\mathrm{CD} 4^{+} \mathrm{T}$ cells. Again, the most frequent naive CD8 clones also had high overlap with the most frequent memory CD8 clones (Figure $5 \mathrm{~F}$ ), consistent with the observation that $\mathrm{CD}^{+}$Tem are more likely to assume a naive-like phenotype than $\mathrm{CD} 4^{+} \mathrm{T}$ cells, as suggested by $\mathrm{CD} 95$ expression within the $\mathrm{CD} 45 \mathrm{RA}^{+} \mathrm{CCR} 7^{+}$compartment. 
To better quantify the TCR overlaps between the compartment of naive and memory cells, we developed a statistical method that provides a metric of how much specific repertoires overlap, either between different subpopulations (memory to naive [pMN]) or between replicates of the same subpopulation (naive to naive $[\mathrm{pNN}]$ and memory to memory $[\mathrm{pMM}]$ ). Using these metrics, we found no statistical difference in the pMN of $\mathrm{CD}^{+} \mathrm{T}$ cells between patients and controls; however, $\mathrm{CD} 8^{+} \mathrm{T}$ cells had a decrease in pMN in patients (Figure 5G), implying that the overlap between the naive and memory CD 8 compartments of patients is slightly less than that in controls. A decrease in $\mathrm{pNN}$ was also observed in patient $\mathrm{CD}^{+} \mathrm{T}$ cells but not in $\mathrm{CD}^{+} \mathrm{T}$ cells (Figure $5 \mathrm{H}$ ) and is consistent with the trend seen for increased TCR richness (Figure $5 \mathrm{~A}$ ). No difference in pMM was observed in either $\mathrm{CD}^{+}$or $\mathrm{CD} 8^{+} \mathrm{T}$ cells (Figure $5 \mathrm{I}$ ). CD8 pMN highly correlated with CD8 pNN, indicating that clonally expanded "naive" T cells are likely reverted naive-like memory cells (Figure 5J). Taken together, these data indicate a diverse regeneration, which consists of an unbiased contraction and reexpansion, of both the naive CD4 and CD8 repertoire. Moreover, it suggests that the naive-like memory cell expansion present within the naive $\mathrm{CD} 8^{+} \mathrm{T}$ cell compartment of patients is not from a single clonotype.

$B$ cell and monocyte repopulation after chemotherapy. Along with $\mathrm{T}$ cell repertoire changes, the high-dimensional comparisons in Figure 1 revealed alterations in $\mathrm{B}$ cell and monocyte populations of patients. Further analysis reveals that $\operatorname{IgD}^{+}$memory $\left(\operatorname{IgD}^{+} \mathrm{CD} 27^{+}\right)$B cell populations were largely depleted, with a trending decrease in class-switched memory $\left(\operatorname{IgD}-\mathrm{CD} 27^{+}\right)$and a trending increase in naive $\left(\operatorname{IgD}{ }^{+} \mathrm{CD} 27^{-}\right)$ B cells (Figure 6A). Although previous studies found increased transitional B cells out to 9 months after chemotherapy (3), we found no difference in transitional B cells between patients and controls, implying that the initial burst of new B cells from the BM wanes within 1 year. Interestingly, the proinflammatory and autoreactive memory population termed "age-associated B cells" (ABCs) was also decreased in patients (Figure $6 \mathrm{~B}$ and refs. 27, 28). These data are consistent with the interpretation that memory B cells are highly susceptible to chemotherapy-induced cell death, with a majority of these cells lost during treatment. Moreover, they imply that recovery of the memory B cell population requires the development of new memory cells from the naive compartment, which has significant implications for antibody-based immune protection and vaccination.

The monocyte compartment displayed modest changes in patients. This population is composed of multiple phenotypic subsets defined by surface marker expression: classical $\left(\mathrm{CD} 14^{++} \mathrm{CD} 16^{-}\right)$, intermediate $\left(\mathrm{CD} 14^{++} \mathrm{CD} 16^{+}\right)$, and nonclassical $\left(\mathrm{CD} 14^{+/ 10} \mathrm{CD} 16^{+}\right)$(Figure $\left.6 \mathrm{C}\right)$. Total monocyte frequencies in patients only trended lower. However, the phenotypic composition was clearly different, with a significant decrease in the frequencies of intermediate monocytes in patients compared with controls (Figure 6D). Decreases in intermediate but not nonclassical monocytes suggest that chemotherapy may modestly affect extravasation and/or lifespan but not differentiation potential of this subset.

Longitudinal impact of chemotherapy on recovery of immune cell composition. Data shown so far were derived from our entire cohort of patients 1-5 years after chemotherapy. To better understand whether observed changes persisted across this entire time period, we investigated the impact of the number of years after chemotherapy treatment on cellular recovery in patients. To determine which of the lymphocyte subsets resolve during this longer time frame, we performed separate scaffold analyses comparing early patients (1-3 years; $n=10$ ) or late patients ( $>3$ years; $n=5$ ) with their chemotherapy-naive, age-matched controls. Within the B cell compartment, we found 3 increased clusters within naive cells present in both early and late patients compared with controls (Figure 7A). However, decreased clusters within the memory B cell compartments were only present in early postchemotherapy comparisons. Further analysis by hand-gating revealed that the $\operatorname{IgD}{ }^{+} \mathrm{CD} 27^{+}$memory population, which is decreased in frequencies in patients $1-3$ years after chemotherapy, is restored in patients 3 or more years after chemotherapy (Figure 7B). Similar results were observed with ABCs. Thus, naive B cells trended to remain higher in patients compared with controls, while decreases in the memory B cell compartment resolved within 3 years after chemotherapy, indicating the conversion of naive to memory cells during this time period. Although recovering in numbers, the antigen specificity repertoire of the new memory B cells remains unknown.

Unlike B cells, patterns of cluster differences within the $\mathrm{CD}^{+} \mathrm{T}$ cell compartment did not resolve between early and later time points (Figure 7C), including patterns highlighted by increased frequencies of Th17 and Th1/17 clusters, as well as decreased naive cell clusters. Hand-gating of the CD4 compartment showed that increased frequencies of Th17 and CD $161^{+} \mathrm{CD} 4^{+}$Tem were maintained in both early and late patient groups compared with controls (Figure 7D); thus, the expansion of $\mathrm{CD} 161^{+}$memory cells 
A

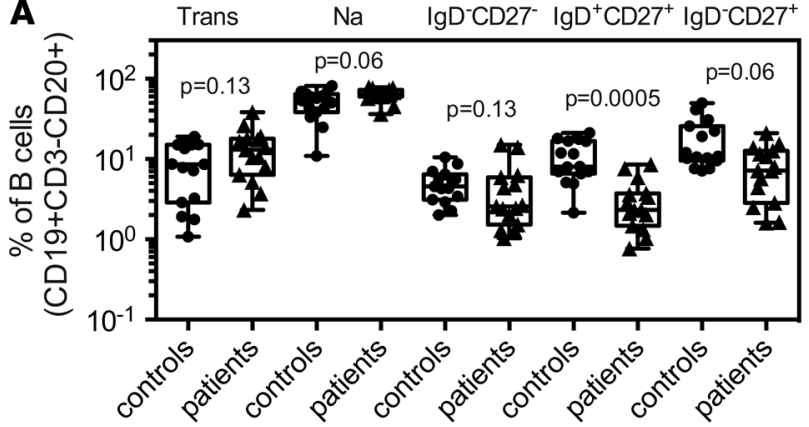

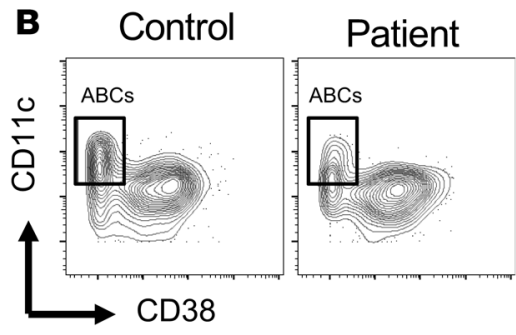

Age-associated $B$ cells $(A B C s)=$ $\operatorname{lgD}-\mathrm{CD} 27-\mathrm{CD} 11 \mathrm{C}+\mathrm{CD} 38-$
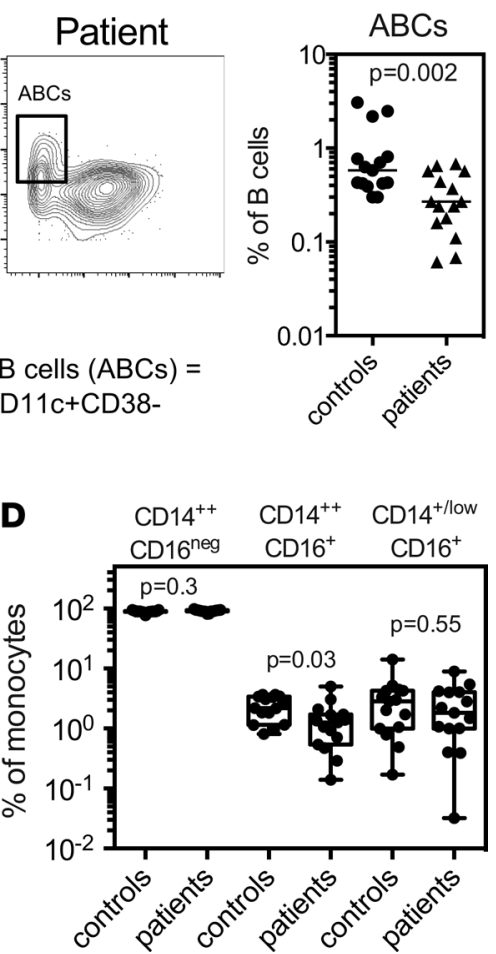

Figure 6. Loss of B cell memory and intermediate monocytes with expansion of naive B cells in patients after chemotherapy. (A) Frequencies of $B$ cell subsets in patients $(n=15)$ and healthy controls $(n=15)$. $P$ values determined by Mann-Whitney $U$ tests with Hochberg multiple comparisons. (B) Frequencies of age-associated B cells (ABC) in patients $(n=15)$ and healthy controls $(n=15)$. $P$ values determined by Mann-Whitney $U$ test. (C) Gating strategy for monocyte subsets: classical (CD14 $\left.{ }^{++} \mathrm{CD} 16^{-}\right)$, intermediate $\left(\mathrm{CD} 14^{++} \mathrm{CD} 16^{+}\right)$, and nonclassical $\left(\mathrm{CD} 14^{+/ 10} \mathrm{CD} 16^{+}\right)$. (D) Frequencies of monocyte subsets in chemotherapy-treated patients $(n=15)$ and healthy controls $(n=15)$. $P$ values determined by Mann-Whitney $U$ tests with Hochberg multiple comparisons.

is persistent in chemotherapy-treated patients. Similar long-term decreases in the naive population were observed (not shown). In order to distinguish the role of cancer versus chemotherapy treatment on CD161 ${ }^{+}$ memory expansion, we analyzed CyTOF data from a small cohort of breast cancer survivors who had not received chemotherapy (surgery and/or radiation only) (29). In contrast to chemotherapy-treated patients, expansion of $\mathrm{CD} 161^{+} \mathrm{CD} 4^{+}$Tem and decreases in naive $\mathrm{CD} 4^{+} \mathrm{T}$ cells were not detected in the breast cancer survivors who had not received chemotherapy (Supplemental Figure 4). Thus, adjuvant chemotherapy causes a long-term persistence of $\mathrm{CD} 161^{+} \mathrm{CD} 4^{+} \mathrm{Tem}$ in patients that could influence subsequent responses to pathogenic infection and vaccination.

\section{Discussion}

Here, we describe the immune cell composition and TCR diversity in breast cancer patients who are at least 1 year past the completion of adjuvant chemotherapy treatment. High-dimensional mass cytometry revealed that most peripheral immune cell subsets exhibit robust recovery within a year. However, the $\mathrm{CD}^{+} \mathrm{T}$ cell compartment displayed significant and persistent alterations in composition, highlighted by incomplete regeneration of naive cells in tandem with a substantial expansion of Th17-like $\mathrm{CD}_{161^{+}}$memory cells. Conversely, the CD8 compartment had relatively rapid and complete repopulation of both naive and memory compartments, with only a modest expansion of $\mathrm{CD}^{2} 5^{+}$cells in the naive compartment. Compositional changes were not mirrored by TCR repertoire contractions, as naive and memory populations in both $\mathrm{CD}^{+}$and $\mathrm{CD}^{+} \mathrm{T}$ cells showed similar TCR diversity as agematched controls. Although the B cell compartment displayed short-term loss of memory cells, these changes resolved within 3-5 years. Conversely, the altered composition of the $\mathrm{CD} 4{ }^{+} \mathrm{T}$ cell compartment was consistently present in all patients out to 5 years after chemotherapy. Thus, our studies reveal that adjuvant chemotherapy has a short-term influence on B cells and a long-lasting, and potentially irreversible, impact on naive and $\mathrm{CD} 4^{+}$Tem that may cause altered immune responses to infection and to vaccination in this growing population of individuals. 
A

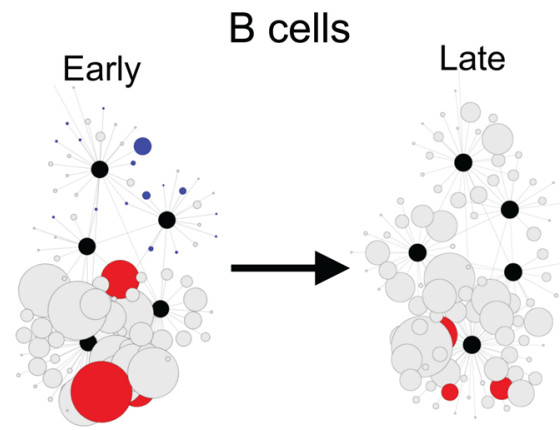

red $=$ increased in patients, blue $=$ decreased in patients, gray = No change, black $=$ landmark node

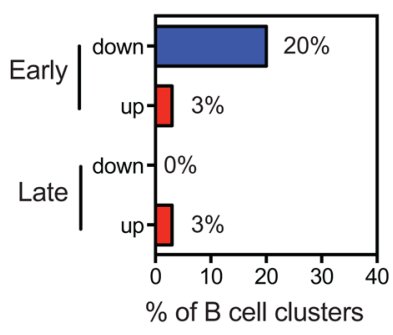

B
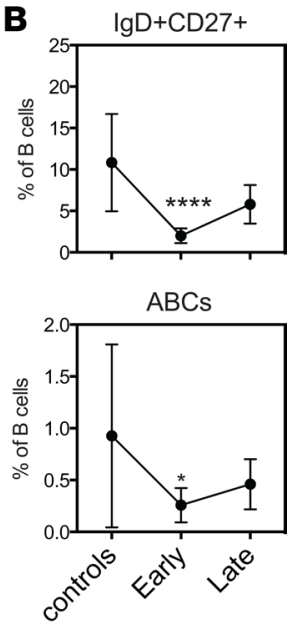

D
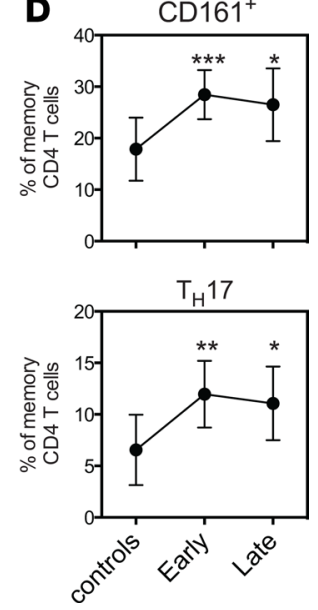

Figure 7. Persistent alterations in the CD4 ${ }^{+} \mathrm{T}$ cell compartment of patients after chemotherapy. (A) Representative scaffold analysis and the percent of differential clusters in B cells between patients $<3$ (early) and 3-5 years (late) after chemotherapy compared with healthy controls. (B) Frequencies of B cell subsets in controls, early patients, and late patients determined by hand-gating. (C) Representative scaffold analysis and the percent of differential clusters in $\mathrm{CD}^{+} \mathrm{T}$ cell compartment cells between patients $<3$ (early) and 3-5 years (late) after chemotherapy compared with healthy controls. Cluster size is proportional to cell frequencies in the individual patient. (D) Frequencies of CD4+ $T$ cell subsets in controls, early patients, and late patients determined by hand-gating. $P$ values were determined by 1-way ANOVA with Tukey's multiple comparison test. Data are shown as mean \pm SD. ${ }^{*} P<0.05 ;{ }^{* *} P<0.01$; ${ }^{* *} P<0.001$; ${ }^{* * * *} P<0.0001$.

Overall, the human immune system has a surprisingly robust capacity to regenerate after lymphopenia, such as that induced by adjuvant chemotherapy. Indeed, our cohort of breast cancer patients recovered in not only their total lymphocyte counts, but also the general compositional complexity of most major innate and adaptive immune cell subsets within the periphery by 1 year after treatment. Innate and adaptive immune cells derive from common hematopoietic stem cells (HSCs) that differentiate into 2 distinct precursor populations within the BM; myeloid (e.g., precursors of monocytes, DCs) and lymphoid (e.g., precursors of T cells, B cells, NK cells) progenitor cells. Due to the quiescent nature of HSCs, this population is naturally more chemotherapy resistant than other, more proliferative precursor cell populations (30). Age-related skewing of HSC differentiation toward a myeloid precursor, as well as thymic involution, would imply that cohorts of older chemotherapy patients such as ours would be inclined toward particularly poor recovery of the adaptive immune system; however, our data demonstrate that these general populations rebound quite nicely. Indeed, B cells even increase in frequencies. Moreover, the T cell rebound was partially driven by increased thymic activity; displayed no evidence of expansion of exhausted or senescent $\mathrm{T}$ cell subsets, such as terminal differentiated effector cells; and maintained extensive TCR diversity. Thus, many features of both the innate and adaptive immune systems can be recovered within a short time period ( $\sim 1$ year) after chemotherapy in breast cancer patients. Thus, for the most part, quantity and quality of immune responses in patients are likely preserved. 
One of the key delineating features of the adaptive immune responses is the ability of $\mathrm{T}$ and $\mathrm{B}$ cells to acquire and maintain antigenic "memory." Previous studies have found that breast cancer patients exhibit reduced serum titers of vaccine-specific antibodies against both T cell-dependent (e.g., tetanus toxoid) and $\mathrm{T}$ cell-independent (e.g., pneumococcal polysaccharide) antigens for at least 9 months after chemotherapy (3). The loss of vaccine-specific antibody responses upon B cell depletion is also well described in studies on the effects of rituximab (CD20 depletion therapy) in rheumatoid arthritis, in which patients treated with rituximab display significantly reduced antibody titers and weaker responses to influenza vaccination than untreated controls (31-33). Notably, AML patients demonstrated significant reduction in somatically hypermutated sequences in the B cell repertoire close to the end of chemotherapy treatment that slowly increased with time after the end of treatment (34), indicative of an initial loss and slow rebound of high-affinity memory B cell responses. The short-term loss of multiple memory B cell subpopulations that, in our study, recovered by 3 years after chemotherapy similarly indicates that memory B cell regeneration occurs via naive $\mathrm{B}$ cells converting to memory upon antigen exposure and not memory cells clonally expanding to fill the depleted niche. Moreover, these data indicate that the newly generated memory B cells would have a substantially different specificity repertoire. The almost complete depletion of $\mathrm{IgD}^{+}$memory B cells early on in patients likely affects their immune protection against mucosal pathogens, as these cells are critical in rapid secondary immune responses through the secretion of high-affinity $\operatorname{IgM}(35,36)$. Clinically, these data imply that revaccination of patients during the 1- to 3-year postchemotherapy window may be needed to rebuild a robust, high-affinity memory B cell repertoire and, thus, provide better immunological protection against pathogenic infection in these individuals.

In order for the generation of Tem targeting new pathogens or new variants of previously seen pathogens, a large, diverse repertoire of naive $\mathrm{T}$ cells is required. Although recovery in naive $\mathrm{T}$ cell numbers was slow and - at least for $\mathrm{CD}^{+} \mathrm{T}$ cells - incomplete, we surprisingly found that naive $\mathrm{T}$ cell populations retained high TCR diversity. Repopulation of naive T cells in lymphopenic conditions is driven by 2 distinct mechanisms; new generation within the thymus and homeostatic proliferation of existing naive cells. Age-related low thymic output is moderately increased after chemotherapy (37), as confirmed in our study. However, the increase was equal for both $\mathrm{CD}^{+}$and $\mathrm{CD} 8^{+} \mathrm{T}$ cells from our patients, suggesting that the contribution was small and does not account for the better recovery of naive $\mathrm{CD} 8^{+}$than $\mathrm{CD} 4^{+} \mathrm{T}$ cells. An altered rate of homeostatic proliferation is the more-likely driver of $\mathrm{CD}^{+}$and $\mathrm{CD} 8^{+}$naive repopulation differences. Studies in mouse models of lymphopenia-induced repopulation have demonstrated that naive $\mathrm{CD}^{+} \mathrm{T}$ cell expansion is significantly slower than that of naive $\mathrm{CD}^{+} \mathrm{T}$ cells $(38-40)$. The preferential reductions in CD4 expansion appears to be at least partially due to reduced expression of MHC-II on antigen-presenting cells, which limits the ability of $\mathrm{CD} 4^{+}$but not $\mathrm{CD} 8^{+} \mathrm{T}$ cells to proliferate (39). Moreover, it has recently been shown that Tem outcompete naive $\mathrm{T}$ cells within tissue niches during lymphopenia-induced repopulation (40), suggesting that full recovery of a naive compartment is unlikely without significant thymopoiesis. In humans, aged naive $\mathrm{CD}^{+} \mathrm{T}$ cells demonstrate higher rates of cell proliferation than in young individuals who may be driven by a slowly accumulating lymphopenia-like environment with age (41). This faster homeostatic proliferation rate of naive $\mathrm{CD} 8^{+} \mathrm{T}$ cells may also induce differentiation and thereby account for the higher expression of CD95 we observed in patients' naive CD8 ${ }^{+} \mathrm{T}$ cell populations.

One of the most striking and long-lasting features in our breast cancer cohort was the expansion of circulating $\mathrm{CD} 161^{+} \mathrm{CD} 4^{+}$Tem This expansion is specifically linked to adjuvant chemotherapy treatment, as chemotherapy-naive, breast cancer patients did not exhibit similar increases in this population. $\mathrm{CD} 161^{+} \mathrm{CD} 4^{+}$ $\mathrm{T}$ cells are a proinflammatory subset highly associated with multiple autoimmune diseases, such as Crohn's disease and rheumatoid arthritis, as well as kidney transplant rejection (42-45). A recent study found CD161 expression, particularly on $\mathrm{CD}^{+} \mathrm{T}$ cells, to be a robust biomarker of improved clinical outcomes in lung cancer patients (46). Tumor-specific CD161 expression is also positively correlated with better survival outcomes for breast, endometrial, and thyroid cancer (www.proteinatlas.org) (47). Notably, CD $161^{+} \mathrm{CD} 4^{+} \mathrm{T}$ cells are uniquely resistant to Treg suppression (43) and have a high propensity to efflux chemotherapy drugs (48), suggesting that they may play an important functional role in tumor clearance and remission. The fact that this cell population remains expanded for more than 5 years in patients similarly indicates active maintenance of this cell population during cancer remission. However, the persistence of these cells is unlikely antigen driven, as we found no increase in clonal expansion within the $\mathrm{CD} 4^{+}$Tem compartment of patients. CD161 expression is induced by tissue-specific factors, such as retinoic acid (49); thus, an alternative explanation for the increases in $\mathrm{CD} 161^{+} \mathrm{CD} 4^{+} \mathrm{T}$ cells is tissue-specific microenvironment changes induced after chemotherapy. 
Determining the drivers of CD161 on $\mathrm{CD} 4^{+}$Tem in chemotherapy-treated breast cancer patients, such as lowgrade tissue inflammation, as well as the functional importance of $\mathrm{CD} 161^{+} \mathrm{CD} 4^{+} \mathrm{T}$ cells in cancer clearance and long-term tumor suppression warrants further investigation.

Overall, our studies have several conclusions with clinical impact. Firstly, CD8 ${ }^{+} \mathrm{T}$ cell immunity appears to be largely preserved; therefore, latent viral infection such as CMV should be well controlled in patients. One possible exception could be varicella zoster virus, as $\mathrm{CD} 4^{+} \mathrm{T}$ cell immunity is essential for preventing reactivation (50). $\mathrm{CD}^{+} \mathrm{T}$ cell responses to newly encountered viruses such as West Nile or Zika virus may also be impaired, due the contraction of the naive compartment. The shift in $\mathrm{CD}^{+}$ Tem composition toward more inflammatory populations should exacerbate a trend that is generally seen with normal aging $(51,52)$ and could contribute to accelerated inflammaging. In addition, this inflammatory bias can impair the generation of $\mathrm{T}$ follicular cells in favor of effector cells (52) and, therefore, reduce the induction of effective B cell memory responses. Similarly, the decline in memory B cells we observed is short-term but severe, and it is highly likely that the regenerated B cell receptor repertoire is not sufficiently protective. Thus, broad revaccinations are desirable not only to produce high antibody titers that are maintained by plasma cells, but more so to generate B cells that can undergo more affinity selection and acquire better antigen recognition upon reinfection. Finally, new vaccination strategies may have to be developed to improve $\mathrm{CD}^{+} \mathrm{T}$ cell responses and compensate for the preferential generation of effector rather than follicular helper cells.

\section{Methods}

Patient cohort. Female breast cancer patients were recruited from the Stanford Cancer Center between 2015 and 2016 based on the following inclusion criteria: female, older than 45 years of age, cancer treated with adjuvant chemotherapy, at least 1 year since receiving the a final of chemotherapy, and in full remission at the time of recruitment. Clinical information on age at the time of blood draw, ethnicity, chemotherapy regimen received, and time since the completion of chemotherapy is provided in Supplemental Table 1, as well as which patient samples were used in each experiment. Age- and sex-matched controls who have never had cancer nor received chemotherapy were recruited locally through Stanford University and the Stanford Blood Center.

Sample collection and subset purification. Whole blood was collected by phlebotomy. PBMCs were isolated by Ficoll density centrifugation. Cells were frozen and stored in liquid nitrogen until use. For TREC analysis and TCR sequencing (see below), naive $\left(\mathrm{CD} 45 \mathrm{RA}^{+} \mathrm{CCR} 7^{+} \mathrm{CD} 28^{+}\right)$and memory $\left(\mathrm{CD} 45 \mathrm{RA}^{-}\right.$and $\mathrm{CD}^{2} 5 \mathrm{RA}^{+} \mathrm{CCR} 7^{-} \mathrm{T}_{\mathrm{EMRA}}$ ) $\mathrm{CD}^{+}$and $\mathrm{CD}^{+} \mathrm{T}$ cells were isolated by cell sorting (FACSAria, BD Biosciences) using the following antibody panel: CD3-Pe/Cy7 (BioLegend, clone UCHT1), CD4-PerCp/Cy5.5 (BioLegend, clone SK3), CD8-APC/Cy7 (BioLegend, clone RPA-T8), CD45RA-FITC (BD Biosciences, clone HI100), CD197 (CCR7)-BV421 (BioLegend, clone G043H7), and CD28-PE (BD Biosciences, clone CD28.2). Dead cells were excluded using LiveDead-eF510 (eBioscience).

Mass cytometry. CyTOF was performed in the Human Immune Monitoring Center at Stanford University. Briefly, PBMCs were thawed in warm media, resuspended in CyFACS buffer (PBS supplemented with $2 \%$ BSA, 2 mM EDTA, and $0.1 \%$ sodium azide), and stained with isotope-tagged antibodies before being acquired on the CyTOF. Antibody details are provided in Supplemental Table 3. In addition, cells were stained with iridium-containing DNA intercalator (DVS Science) and Live-Dead stain (DOTA-maleimide [Macrocyclics] containing natural-abundance indium) for determining live, single cells. A detailed staining protocol can be found at http://iti.stanford.edu/himc/protocols.html under "CyTOF Immunophenotyping." All samples were run with normalization beads to allow signal normalization across CyTOF runs. Samples were run in 2 batches, with each patient paired with their respective healthy control as an internal control. Raw data acquired from CyTOF were first normalized using premessa (http://github.com/ParkerICI/premessa; commit ID https://github.com/ParkerICI/ premessa/releases/tag/v0.1.8). Normalized data were analyzed using FlowJo v10.1 (FlowJo Inc.) by hand-gating (Supplemental Figures 1 and 2). Selected populations (e.g., live cells, CD4 ${ }^{+} \mathrm{T}$ cells, CD8 ${ }^{+} \mathrm{T}$ cells) were then exported for downstream analysis.

Statistical scaffold analysis. Clustering and population comparisons were performed on indicated cell populations using the open source R package Statistical Scaffold (http://github.com/SpitzerLab/ statisticalScaffold) (18). In brief, individual files from each subject/control were uploaded and clustered using asinh cofactor of 5 . Specific parameters (cluster number, sample number, and markers used 
for clustering) for cell clustering are provided in Supplemental Table 4. After clustering, frequency statistics between chemotherapy-naive, age-matched controls (group 1) and patients (group 2) were determined using a $Q$ value cutoff of 5 , which sets the threshold of significance at $5 \%$, and 10,000 permutations. The $Q$ values are determined using significance analysis of microarrays as described in Spitzer et al. (18) and are corrected for multiple comparisons. Scaffold maps were then generated using the same markers as selected for clustering with concatenated hand-gated cell populations from the 15 control samples used as landmark nodes, with a proportional edge-weight influence and asinh cofactor of 5 . In all scaffold maps, a minimum node size of 1 , maximum node size of 100 , and a landmark node size of 10 were used. Clusters with $Q$ values determined to be significant $(Q<0.05)$ are colored either red or blue depending on directionality.

TREC analysis. DNA was isolated from sorted naive $\mathrm{CD}^{+}$and $\mathrm{CD}^{+} \mathrm{T}$ cells using Qiagen AllPrep DNA/RNA extraction kit. TRECs were analyzed using MyTREC RealTime qPCR assay kit (Genenplus).

$T$ cell receptor sequencing. Sorted naive $\left(\mathrm{CD} 45 \mathrm{RA}{ }^{+} \mathrm{CCR} 7^{+} \mathrm{CD} 28^{+}\right)$and memory $\left(\mathrm{CD} 45 \mathrm{RA}^{-}\right.$and $\left.\mathrm{CD} 4 \mathrm{RA}^{+} \mathrm{CCR} 7^{-}\right) \mathrm{CD}^{+}$and $\mathrm{CD}^{+}{ }^{+} \mathrm{T}$ cells were sequenced as previously described (26). Briefly, RNA was extracted from 5 replicates of each subset (Qiagen All Prep DNA/RNA Kit). For CD4 ${ }^{+} \mathrm{T}$ cells, there were 200,000-250,000 cells in each replicate. For CD8 ${ }^{+}$T cells, there were 100,000-200,000 cells per replicate. cDNA was synthesized (VILO mastermix, Invitrogen) and TRB genes were barcoded, amplified and Illumina sequencing adapters were added using 2 rounds of PCR (Qiagen multiplex PCR plus kit). Sequencing was performed on a miSeq (Illumina).

$T C R$ repertoire analysis. The reads obtained from miSeq were debarcoded after trimming adaptors. The reads were then aligned to the human TRB reference sequence library from IMGT (January 2017) (53) and assembled into clones using MiXCR v2.1.2 (54). These clones were subsequently used for downstream analyses using tcR package (55). Circos plots were generated by adapting the implementation from the ClonoPlot package (https://doi.org/10.1186/s12859-017-1575-2), where clone overlaps were determined by matching clones with similar CDR3 amino acid sequences. Clonality and richness of the repertoire was done using the lymphclon package from $\mathrm{R}$ and Chao2 nonparametric estimator, respectively, as described previously by our group (26). We used CDR3 nucleotide sequence similarity to determine the probability of clone overlaps within replicates and across memory and naive subsets.

To quantify the amount of overlap between populations, we developed the following metrics using amino acid CDR3 sequence for determining clones: (a) pMN, which is the probability that a randomly selected naive $\mathrm{T}$ cell and a randomly selected memory $\mathrm{T}$ cell are from the same clone; (b) pNN, which is the probability that a randomly selected naive $\mathrm{T}$ cell from 1 replicate and a randomly selected naive $\mathrm{T}$ cell from a second replicate are from the same clone; and (c) pMM, which is the probability that a randomly selected memory $\mathrm{T}$ cell from 1 replicate and a randomly selected memory $\mathrm{T}$ cell from a second replicate are from the same clone. The $\mathrm{R}$ code for calculating these probabilities is provided in the Supplemental Methods.

The accession number for TRB sequencing data reported in this paper is SRA PRJNA598860. The mass cytometry data supporting this publication is available at ImmPort (http://www.immport.org) under study accession SDY1597.

Statistics. Data were analyzed using Mann-Whitney $U$ test, Kruskall-Wallis test with Dunn's multiple comparison or 1-way ANOVA with Tukey's multiple comparison test as appropriate and as indicated in the specific figure legends. Multiple comparison analysis was performed using Hochberg multiple comparison, which controls for family-wise type 1 error within each set of the analyses. Statistical tests were performed using GraphPad Prism version 6. Multiple comparison testing was performed in R using p.adjust function. $P<0.05$ is considered statistically significant.

Study approval. Studies were in accordance with the Declaration of Helsinki and approved by Stanford University IRB. All participants gave written informed consent before inclusion in these studies.

\section{Author contributions}

CEG recruited donors, designed and performed experiments, and analyzed data. RJ performed TCR sequencing data analysis. WC and QQ performed experiments. MP recruited donors, participated in study design, and provided critical review of the manuscript. LT developed computational methods. CMW and JJG provided supervision and designed experiments. CEG and JJG wrote the manuscript. 


\section{Acknowledgments}

We thank the Human Immune Monitoring Core at Stanford University — in particular, Michael Leipold and Holden Maecker - for mass cytometry services. This work was supported by the NIH (R01 AI108891, R01 AG045779, U19 AI057266, U19 AI057229, and R01 AI129191 to JJG; R01 AR042527, R01 HL117913, R01 AI108906, R01 HL142068, and P01 HL129941 to CMW; and T32 AG047126 to CEG), by the Breast Cancer Research Foundation (to MP), and with resources and the use of facilities at the Palo Alto Veterans Administration Healthcare System. CEG is an Irene Diamond Fund/AFAR Award recipient.

Address correspondence to: Jörg J. Goronzy, Stanford University, CCSR Building Room 2225, 269 Campus Drive West, Stanford, California 94305-5166, USA. Phone: 650.723.9027; Email: jgoronzy@stanford.edu.

1. Mackall CL, et al. Lymphocyte depletion during treatment with intensive chemotherapy for cancer. Blood. 1994;84(7):2221-2228.

2. Tao JJ, Visvanathan K, Wolff AC. Long term side effects of adjuvant chemotherapy in patients with early breast cancer. Breast. 2015;24 Suppl 2:S149-S153.

3. Verma R, et al. Lymphocyte depletion and repopulation after chemotherapy for primary breast cancer. Breast Cancer Res. 2016;18(1):10.

4. Fagnoni FF, et al. T-cell dynamics after high-dose chemotherapy in adults: elucidation of the elusive CD8+ subset reveals multiple homeostatic T-cell compartments with distinct implications for immune competence. Immunology. 2002;106(1):27-37.

5. Havenith SH, et al. Rapid T cell repopulation after rabbit anti-thymocyte globulin (rATG) treatment is driven mainly by cytomegalovirus. Clin Exp Immunol. 2012;169(3):292-301.

6. Mackall CL, et al. Distinctions between CD8+ and CD4+ T-cell regenerative pathways result in prolonged T-cell subset imbalance after intensive chemotherapy. Blood. 1997;89(10):3700-3707.

7. Pang WW, et al. Human bone marrow hematopoietic stem cells are increased in frequency and myeloid-biased with age. Proc Natl Acad Sci USA. 2011;108(50):20012-20017.

8. Rossi DJ, et al. Cell intrinsic alterations underlie hematopoietic stem cell aging. Proc Natl Acad Sci USA. 2005;102(26):9194-9199.

9. Bains I, Antia R, Callard R, Yates AJ. Quantifying the development of the peripheral naive CD4+ T-cell pool in humans. Blood. 2009;113(22):5480-5487.

10. Hakim FT, et al. Age-dependent incidence, time course, and consequences of thymic renewal in adults. J Clin Invest. 2005;115(4):930-939.

11. Surh CD, Sprent J. Homeostasis of naive and memory T cells. Immunity. 2008;29(6):848-862.

12. Hamilton SE, Wolkers MC, Schoenberger SP, Jameson SC. The generation of protective memory-like CD8+ T cells during homeostatic proliferation requires CD4+ T cells. Nat Immunol. 2006;7(5):475-481.

13. Pribikova M, Moudra A, Stepanek O. Opinion: Virtual memory CD8 T cells and lymphopenia-induced memory CD8 T cells represent a single subset: Homeostatic memory T cells. Immunol Lett. 2018;203:57-61.

14. Quinn KM, et al. Age-Related Decline in Primary CD8 ${ }^{+} \mathrm{T}$ Cell Responses Is Associated with the Development of Senescence in Virtual Memory CD8 ${ }^{+}$T Cells. Cell Rep. 2018;23(12):3512-3524.

15. White JT, et al. Virtual memory T cells develop and mediate bystander protective immunity in an IL-15-dependent manner. Nat Commun. 2016;7:11291.

16. Looney RJ, et al. Role of cytomegalovirus in the T cell changes seen in elderly individuals. Clin Immunol. 1999;90(2):213-219.

17. Weinberger B, et al. Healthy aging and latent infection with CMV lead to distinct changes in CD8+ and CD4+ T-cell subsets in the elderly. Hum Immunol. 2007;68(2):86-90.

18. Spitzer MH, et al. Systemic Immunity Is Required for Effective Cancer Immunotherapy. Cell. 2017;168(3):487-502.e15.

19. Wertheimer AM, et al. Aging and cytomegalovirus infection differentially and jointly affect distinct circulating $\mathrm{T}$ cell subsets in humans. J Immunol. 2014;192(5):2143-2155.

20. Mahnke YD, Brodie TM, Sallusto F, Roederer M, Lugli E. The who's who of T-cell differentiation: human memory T-cell subsets. Eur J Immunol. 2013;43(11):2797-2809.

21. Kunicki MA, Amaya Hernandez LC, Davis KL, Bacchetta R, Roncarolo MG. Identity and Diversity of Human Peripheral Th and T Regulatory Cells Defined by Single-Cell Mass Cytometry. J Immunol. 2018;200(1):336-346.

22. Fuertes Marraco SA, et al. Long-lasting stem cell-like memory CD8+ T cells with a naïve-like profile upon yellow fever vaccination. Sci Transl Med. 2015;7(282):282ra48.

23. Gattinoni L, et al. A human memory T cell subset with stem cell-like properties. Nat Med. 2011;17(10):1290-1297.

24. Pulko V, et al. Human memory T cells with a naive phenotype accumulate with aging and respond to persistent viruses. Nat Immunol. 2016;17(8):966-975.

25. Maggi L, et al. CD161 is a marker of all human IL-17-producing T-cell subsets and is induced by RORC. Eur J Immunol. 2010;40(8):2174-2181.

26. Qi Q, et al. Diversity and clonal selection in the human T-cell repertoire. Proceedings of the National Academy of Sciences of the United States of America. 2014;111(36).

27. Hao Y, O'Neill P, Naradikian MS, Scholz JL, Cancro MP. A B-cell subset uniquely responsive to innate stimuli accumulates in aged mice. Blood. 2011;118(5):1294-1304.

28. Rubtsov AV, et al. Toll-like receptor 7 (TLR7)-driven accumulation of a novel CD11 ${ }^{+}$B-cell population is important for the development of autoimmunity. Blood. 2011;118(5):1305-1315.

29. Whiting CC, et al. Large-Scale and Comprehensive Immune Profiling and Functional Analysis of Normal Human Aging. PLoS ONE. 2015;10(7):e0133627.

30. Nakamura-Ishizu A, Takizawa H, Suda T. The analysis, roles and regulation of quiescence in hematopoietic stem cells. Development. 
2014;141(24):4656-4666.

31. Gelinck LB, Teng YK, Rimmelzwaan GF, van den Bemt BJ, Kroon FP, van Laar JM. Poor serological responses upon influenza vaccination in patients with rheumatoid arthritis treated with rituximab. Ann Rheum Dis. 2007;66(10):1402-1403.

32. van Assen $S$, et al. Humoral responses after influenza vaccination are severely reduced in patients with rheumatoid arthritis treated with rituximab. Arthritis Rheum. 2010;62(1):75-81.

33. Eisenberg RA, et al. Rituximab-treated patients have a poor response to influenza vaccination. J Clin Immunol. 2013;33(2):388-396.

34. Goswami M, et al. Impaired B cell immunity in acute myeloid leukemia patients after chemotherapy. J Transl Med. 2017;15(1):155.

35. Kruetzmann S, et al. Human immunoglobulin M memory B cells controlling Streptococcus pneumoniae infections are generated in the spleen. J Exp Med. 2003;197(7):939-945.

36. Shi Y, Agematsu K, Ochs HD, Sugane K. Functional analysis of human memory B-cell subpopulations: IgD+CD27+ B cells are crucial in secondary immune response by producing high affinity IgM. Clin Immunol. 2003;108(2):128-137.

37. Sun DP, et al. Thymic hyperplasia after chemotherapy in adults with mature B cell lymphoma and its influence on thymic output and CD4(+) T cells repopulation. Oncoimmunology. 2016;5(5):e1137417.

38. Geiselhart LA, Humphries CA, Gregorio TA, Mou S, Subleski J, Komschlies KL. IL-7 administration alters the CD4:CD8 ratio, increases T cell numbers, and increases T cell function in the absence of activation. J Immunol. 2001;166(5):3019-3027.

39. Guimond M, et al. Interleukin 7 signaling in dendritic cells regulates the homeostatic proliferation and niche size of CD4+ T cells. Nat Immunol. 2009;10(2):149-157.

40. Kim HK, et al. Differential Cytokine Utilization and Tissue Tropism Results in Distinct Repopulation Kinetics of Naïve vs Memory T Cells in Mice. Front Immunol. 2019;10:355.

41. Westera L, et al. Lymphocyte maintenance during healthy aging requires no substantial alterations in cellular turnover. Aging Cell. 2015;14(2):219-227.

42. Kim KW, et al. Clinical significance of CD161+CD4+ T cells in the development of chronic antibody-mediated rejection in kidney transplant recipients. PLoS ONE. 2018;13(7):e0200631.

43. Basdeo SA, et al. Polyfunctional, Pathogenic CD161+ Th17 Lineage Cells Are Resistant to Regulatory T Cell-Mediated Suppression in the Context of Autoimmunity. J Immunol. 2015;195(2):528-540.

44. Fergusson JR, et al. CD161 defines a transcriptional and functional phenotype across distinct human T cell lineages. Cell Rep. 2014;9(3):1075-1088.

45. Bai A, et al. CD39 and CD161 modulate Th17 responses in Crohn's disease. J Immunol. 2014;193(7):3366-3377.

46. Braud VM, et al. Expression of LLT1 and its receptor CD161 in lung cancer is associated with better clinical outcome. Oncoimmunology. 2018;7(5):e1423184.

47. Uhlen M, et al. A pathology atlas of the human cancer transcriptome. Science. 2017;357(6352):eaan2507.

48. Alsuliman A, et al. A subset of virus-specific $\mathrm{CD} 161^{+} \mathrm{T}$ cells selectively express the multidrug transporter MDR1 and are resistant to chemotherapy in AML. Blood. 2017;129(6):740-758.

49. Povoleri GAM, et al. Human retinoic acid-regulated CD $161^{+}$regulatory T cells support wound repair in intestinal mucosa. Nat Immunol. 2018;19(12):1403-1414.

50. Haberthur $\mathrm{K}$, et al. CD4 T cell immunity is critical for the control of simian varicella virus infection in a nonhuman primate model of VZV infection. PLoS Pathog. 2011;7(11):e1002367.

51. Fang F, et al. Expression of CD39 on Activated T Cells Impairs their Survival in Older Individuals. Cell Rep. 2016;14(5):1218-1231

52. Kim C, et al. Activation of miR-21-Regulated Pathways in Immune Aging Selects against Signatures Characteristic of Memory T Cells. Cell Rep. 2018;25(8):2148-2162.e5.

53. Lefranc MP. Immunoglobulin and T Cell Receptor Genes: IMGT(®) and the Birth and Rise of Immunoinformatics. Front Immunol. 2014;5:22

54. Bolotin DA, et al. MiXCR: software for comprehensive adaptive immunity profiling. Nat Methods. 2015;12(5):380-381.

55. Nazarov VI, et al. tcR: an R package for T cell receptor repertoire advanced data analysis. BMC Bioinformatics. 2015;16:175. 